\title{
Effect Handlers, Evidently
}

\author{
NINGNING XIE, Microsoft Research, USA \\ JONATHAN IMMANUEL BRACHTHÄUSER, University of Tübingen, Germany \\ DANIEL HILLERSTROM, The University of Edinburgh, United Kingdom \\ PHILIPP SCHUSTER, University of Tübingen, Germany \\ DAAN LEIJEN, Microsoft Research, USA
}

Algebraic effect handlers are a powerful way to incorporate effects in a programming language. Sometimes perhaps even too powerful. In this article we define a restriction of general effect handlers with scoped resumptions. We argue one can still express all important effects, while improving reasoning about effect handlers. Using the newly gained guarantees, we define a sound and coherent evidence translation for effect handlers, which directly passes the handlers as evidence to each operation. We prove full soundness and coherence of the translation into plain lambda calculus. The evidence in turn enables efficient implementations of effect operations; in particular, we show we can execute tail-resumptive operations in place (without needing to capture the evaluation context), and how we can replace the runtime search for a handler by indexing with a constant offset.

\section{CCS Concepts: • Software and its engineering $\rightarrow$ Control structures; Polymorphism; • Theory of} computation $\rightarrow$ Type theory.

Additional Key Words and Phrases: Algebraic Effects, Handlers, Evidence Passing Translation

ACM Reference Format:

Ningning Xie, Jonathan Immanuel Brachthäuser, Daniel Hillerström, Philipp Schuster, and Daan Leijen. 2020. Effect Handlers, Evidently. Proc. ACM Program. Lang. 4, ICFP, Article 99 (August 2020), 29 pages. https://doi.org/10.1145/3408981

\section{INTRODUCTION}

Algebraic effects [Plotkin and Power 2003] and the extension with handlers [Plotkin and Pretnar 2013], are a powerful way to incorporate effects in programming languages. Algebraic effect handlers can express any free monad in a concise and composable way, and can be used to express complex control-flow, like exceptions, asynchronous I/O, local state, backtracking, and many more.

Even though there are many language implementations of algebraic effects, like Koka [Leijen 2014], Eff [Pretnar 2015], Frank [Lindley et al. 2017], Links [Lindley and Cheney 2012], and Multicore OCaml [Dolan et al. 2015], the implementations may not be as efficient as one might hope. Generally, handling effect operations requires a linear search at runtime to the innermost handler. This is a consequence of the core operational rule for algebraic effect handlers:

handle $\mathrm{e}_{m} h \mathrm{E}[$ perform $o p v] \longrightarrow f v k$

Authors' addresses: Ningning Xie, Microsoft Research, USA, nnxie@cs.hku.hk; Jonathan Immanuel Brachthäuser, University of Tübingen, Germany, jonathan.brachthaeuser@uni-tuebingen.de; Daniel Hillerström, The University of Edinburgh, United Kingdom, daniel.hillerstrom@ed.ac.uk; Philipp Schuster, University of Tübingen, Germany, philipp.schuster@unituebingen.de; Daan Leijen, Microsoft Research, USA, daan@microsoft.com.

This work is licensed under a Creative Commons Attribution-NoDerivatives 4.0 International License.

(c) 2020 Copyright held by the owner/author(s).

2475-1421/2020/8-ART99

https://doi.org/10.1145/3408981

Proc. ACM Program. Lang., Vol. 4, No. ICFP, Article 99. Publication date: August 2020. 
requiring that $(o p \rightarrow f)$ is in the handler $h$ and that $o p$ is not in the bound operations in the evaluation context $\mathrm{E}$ (so the innermost handler gets to handle the operation). The operation clause $f$ gets passed the operation argument $v$ and the resumption $k=\lambda x$. handle $m h \mathrm{E}[x]$. The reduction rule suggests that implementations need to search through the evaluation context to find the innermost handler, capture the context up to that point as the resumption, and can only then invoke the actual operation clause $f$. This search often is linear in the size of the stack, or in the number of intermediate handlers in the context $\mathrm{E}$.

In prior work, it has been shown that the vast majority of operations can be implemented more efficiently, often in time constant in the stack size. Doing so, however, requires an intricate runtime system [Dolan et al. 2015; Leijen 2017a] or explicitly passing handler implementations, instead of dynamically searching for them [Brachthäuser et al. 2018; Zhang and Myers 2019]. While the latter appears an attractive alternative to implement effect handlers, a correspondence between handler passing and dynamic handler search has not been formally established in the literature.

In this article, we make this necessary connection and thereby open up the way to efficient compilation of effect handlers. We identify a simple restriction of general effect handlers, called scoped resumptions, and show that under this restriction we can perform a sound and coherent evidence translation for effect handlers. In particular:

- The ability of effect handlers to capture the resumption $k$ as a first-class value is very powerful - perhaps too powerful as it can interfere with the ability to reason about the program. We define the notion of scoped resumptions (Section 2.2) as a restriction of general effect handlers where resumptions can only be applied in the very scope of their original handler context. We believe all important effect handlers can be written with scoped resumptions, while at the same time ruling out many "wild" applications that have non-intuitive semantics. In particular, it rules out handlers that change semantics of other operations than the ones it handles itself. This improves the ability to reason about effects, and the coherence of evidence translation turns out to only be preserved under scoped resumptions (more precisely: an evidence translated program does not get stuck if resumptions are scoped). In this paper, we focus on the evidence translation and use a dynamic check in our formalism. We show various designs on how to check this property statically, but leave full exploration of such a check to future work.

- To open up the way to more efficient implementations, we define a type-directed evidence translation (Section 4) where a vector of handlers is passed down as an implicit parameter to all operation invocations; similar to the dictionary translation in Haskell for type classes [Jones 1992], or capability passing in Effekt [Brachthäuser et al. 2020]. This turns out to be surprisingly tricky to get right, and we describe various pitfalls in Section 4.2. We prove that our translation is sound (Theorem 4 and 7) and coherent (Theorem 8), and that the evidence provided at runtime indeed always corresponds exactly to the dynamic innermost handler in the evaluation context (Theorem 5). In particular, on an evaluation step:

$$
\text { handle }_{m} h \mathrm{E}[\text { perform op ev } v] \quad \longrightarrow f v k \quad \text { with } o p \notin \operatorname{bop}(\mathrm{E}) \wedge(o p \rightarrow f) \in h
$$

the provided evidence $e v$ will be exactly the pair $(m, h)$, uniquely identifying the actual (dynamic) handler $m$ and its implementation $h$. This is the essence to enabling further optimizations for efficient algebraic effect handlers.

Building on the coherent evidence translation, we describe various techniques for more efficient implementations (Section 6):

- In practice, the majority of effects is tail resumptive, that is, their operation clauses have the form $o p \rightarrow \lambda x . \lambda k$. $k e$ with $k \notin \mathrm{fv}(e)$. That is, they always resume once in the end with the operation result. Note that $e$ may use $x$ or perform operations itself, as it has already 
captured (closed over) the specific evidence it needs when the handler was instantiated. We can execute such tail resumptive operation clauses in place, e.g.

$$
\text { perform op }(m, h) v \rightarrow f v(\lambda x . x) \quad\left(o p_{\text {tail }} \rightarrow f\right) \in h
$$

This is an important optimization that enables truly efficient effect operations at a cost similar to a virtual method call (since we can implement handlers $h$ as a vector of function pointers where $o p$ is at a constant offset such that $f=h . o p$ ).

- Generally, evidence is passed as an evidence vector $w$ where each element is the evidence for a specific effect. That means we still need to select the right evidence at run-time which is a linear time operation (much like the dynamic search for the innermost handler in the evaluation context). We show that by keeping the evidence vectors in canonical form, we can index the evidence in the vector at a constant offset for any context where the effect is non-polymorphic.

- Since the evidence provides the handler implementation directly, it is no longer needed in the context. We can follow Brachthäuser and Schuster [2017] and implement handlers using multi-prompt delimited continuations [Dyvbig et al. 2007; Gunter et al. 1995] instead. Given evidence $(m, h)$, we directly yield to a specific prompt $m$ :

$$
\begin{aligned}
& \text { handle }_{m} h \mathrm{E}[\text { perform op }(m, h) v] \\
& \rightsquigarrow \\
& \text { prompt }_{m} \mathrm{E}\left[\text { yield }_{m}(\lambda k .(h . o p) v k)\right]
\end{aligned}
$$

We define a monadic multi-prompt translation (Section 5) from an evidence translated program (in $\mathrm{F}^{e v}$ ) into standard call-by-value polymorphic lambda calculus $\left(\mathrm{F}^{v}\right)$ where the monad implements the multi-prompt semantics, and we prove that this monadic translation is sound (Theorem 10) and coherent (Theorem 11). Such translation is very important, as it provides the missing link between traditional implementations based on dynamic search for the handler [Dolan et al. 2015; Leijen 2014; Lindley et al. 2017] and implementations of lexical effect handlers using multi-prompt delimited control [Biernacki et al. 2019; Brachthäuser and Schuster 2017; Zhang and Myers 2019]. Since all effects become explicit, we can compile programs with a standard backend applying the usual optimizations that would not hold under algebraic effect semantics, directly. For example, as all handlers become regular data types, and evidence is a regular parameter, standard optimizations like inlining can often completely inline the operation clauses at the call site without any special optimization rules for effect handlers [Pretnar et al. 2017]. Moreover, no special runtime system for capturing the evaluation context is needed anymore, such as split-stacks [Dolan et al. 2015] or stack copying [Leijen 2017a], and we can generate code directly for any host platform (including $\mathrm{C}$ or WebAssembly). In particular, recent advances in compilation guided reference counting [Ullrich and Moura 2019] can readily be used. Such reference counting transformations cannot be applied to traditional effect handler semantics since any effect operation may not resume (or resume more than once), making it impossible to track the reference counts directly.

We start by giving an overview of algebraic effects and handlers and their semantics in an untyped calculus $\lambda^{\epsilon}$ (Section 2), followed by a typed polymorphic formalization $F^{\epsilon}$ (Section 3) for which we prove various theorems like soundness, preservation, and the meaning of effect types. In Section 4 we define an extension of $F^{\epsilon}$ with explicit evidence vector parameters, called $F^{e v}$, define a formal evidence passing translation, and prove this translation is coherent and preserves the original semantics. Using the evidence translated programs, we define a coherent monadic translation in Section 5 (based on multi-prompt semantics) that translates into standard call-by-value polymorphic lambda-calculus $\left(\right.$ called $\mathrm{F}^{v}$ ). Section 6 discusses various immediate optimization techniques enabled 


\begin{tabular}{|c|c|c|c|c|c|}
\hline \multicolumn{3}{|c|}{ Expressions } & \multicolumn{3}{|l|}{ Values } \\
\hline$e \quad::=$ & $v$ & (value) & $v \quad::=$ & $x$ & (variables) \\
\hline & e e & (application) & & $\lambda x . e$ & (functions $f$ ) \\
\hline & handle $h e$ & (handling) & & $\begin{array}{l}\text { handler } h \\
\text { perform } o p\end{array}$ & $\begin{array}{l}\text { (effect handler) } \\
\text { (operation) }\end{array}$ \\
\hline
\end{tabular}

$\begin{array}{llll}\text { Handlers } & h::=\left\{o p_{1} \rightarrow f_{1}, \ldots, o p_{n} \rightarrow f_{n}\right\} & \text { (operation clauses) } \\ \text { Evaluation Context } & \mathrm{F}::=\square|\mathrm{F} e| v \mathrm{~F} & \text { (pure evaluation) } \\ & \mathrm{E}::=\square|\mathrm{E} e| v \mathrm{E} \mid \text { handle } h \mathrm{E} & \text { (effectful computation) }\end{array}$

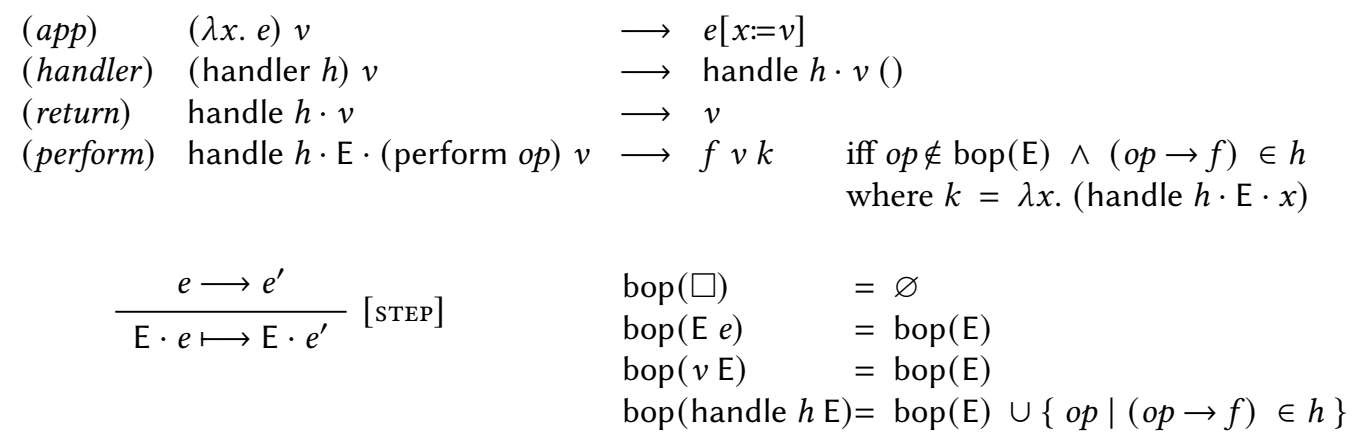

Fig. 1. $\lambda^{\epsilon}$ : Untyped Algebraic Effect Handlers

by evidence passing, in particular tail-resumption optimization, effect-selective monadic translation, and bind-inlining to avoid explicit allocation of continuations.

For space reasons, all evaluation context type rules and the full proofs of all stated lemmas and theorems can be found in a separate extended technical report [Xie et al. 2020], which also includes further discussion of possible extensions.

\section{UNTYPED ALGEBRAIC EFFECT HANDLERS}

We begin by formalizing a minimal calculus of untyped algebraic effect handlers, called $\lambda^{\epsilon}$. The formalization helps introducing the background, sets up the notations used throughout the paper, and enables us to discuss examples in a more formal way.

The formalization of $\lambda^{\epsilon}$ is given in Figure 1. It essentially is a standard call-by-value lambda calculus extended with syntax to perform operations and to handle them. It corresponds closely to the untyped semantics of Forster et al. [2019], and the effect calculus presented by Leijen [2017c]. Sometimes, effect handler semantics are given in a form that does not use evaluation contexts, e.g. [Kammar and Pretnar 2017; Pretnar 2015], but in the end both formulations are equivalent (except that using evaluation contexts turns out to be convenient for our proofs).

There are two differences to earlier calculi: we leave out return clauses (for simplicity) and instead of one handle $h$ expression we distinguish between handle $h e$ (as an expression) and handler $h$ (as a value). A (handler $h) v$ evaluates to handle $h(v())$ and just invokes its given function $v$ with a unit value under a handle $h$ frame. As we will see later, handler is generative and instantiates handle frames with a unique marker. As such, we treat handle as a strictly internal frame that only occurs during evaluation.

The evaluation contexts consist of pure evaluation contexts $\mathrm{F}$ and effectful evaluation contexts $\mathrm{E}$ that include handle $h \mathrm{E}$ frames. We assume a set of operation names op. The (perform op) $v$ 
construct calls an effect operation op by passing it a value $v$. Operations are handled by handle $h e$ expressions, which can be seen in the (perform) rule. Here, the condition op $\notin$ bop(E) ensures that the innermost handle frame handles an operation. Evaluation continues with the body of the operation clause (op $\rightarrow f$ ), passing the argument value $v$ and the resumption $k$ to $f$. Note that $f v k$ is not evaluated under the handler $h$, while the resumption always resumes under the handler $h$ again; this describes the semantics of deep handlers and correspond to a fold in a categorical sense, as opposed to shallow handlers that are more like a case [Kammar et al. 2013].

For conciseness, we often use dot notation to decompose and compose evaluation contexts, which also conveys more clearly that an evaluation context essentially corresponds to a runtime stack. Dot notation is defined as:

$$
\begin{aligned}
& \mathrm{E} \cdot e \doteq \mathrm{E}[e] \\
& \square e \cdot \mathrm{E} \doteq \mathrm{E} e
\end{aligned}
$$

$$
v \square \cdot \mathrm{E} \quad \dot{=} v \cdot \mathrm{E} \quad \dot{=} v \mathrm{E}
$$

handle $h \square \cdot \mathrm{E} \doteq$ handle $h \cdot \mathrm{E} \doteq$ handle $h \mathrm{E}$

For example, we would write $v \cdot$ handle $h \cdot \mathrm{E} \cdot e$ as a shorthand for $v$ (handle $h(\mathrm{E}[e])$ ).

\subsection{Examples}

Here are some examples of common effect handlers. Many practical uses of effect handlers are a variation of these.

Exception: Assuming we have data constructors just and nothing, we can define a handler for exceptions that converts any exceptional computation $e$ to either just $v$ or nothing:

handler $\{$ throw $\rightarrow \lambda x$. $\lambda k$. nothing $\}\left(\lambda_{-}\right.$. just $\left.e\right)$

For example using $e=$ perform throw () evaluates to nothing while $e=1$ evaluates to just 1 .

Reader: In the exception example we just ignored the argument and the resumption of the operation but the reader effect uses the resumption to resume with a result:

handler $\{$ get $\rightarrow \lambda x . \lambda k . k 1\}\left(\lambda_{-}\right.$. perform get ()$+$perform get ()$)$

Here we handle the get operation to always return 1 so the evaluation proceeds as:

handler $\{$ get $\rightarrow \lambda x . \lambda k . k 1\}\left(\lambda_{-}\right.$. perform get ()$+$perform get ()$)$

$\longmapsto^{*}$ handle $h \cdot$ perform get ()$+$ perform get ()

$\longmapsto^{*}(\lambda x$. handle $h \cdot(\square+$ perform get ()$) \cdot x) 1$

$\longmapsto$ handle $h \cdot(\square+$ perform get ()$) \cdot 1$

$\longmapsto{ }^{*}$ handle $h \cdot(1+\square) \cdot 1$

$\longmapsto^{*} 2$

State: We present three variants of how to encode state using effect handlers. The first variant is quite involved as we return functions from the operation clauses - like the state monad (variant $\mathbf{1}$ ):

$h=\{$ get $\rightarrow \lambda x . \lambda k .(\lambda y . k y y)$, set $\rightarrow \lambda x . \lambda k .(\lambda y . k() x)\}$

(handler $h\left(\lambda_{-}\right.$. (perform set $21 ; x \leftarrow$ perform get ()$\left.\left.;(\lambda y \cdot x+x)\right)\right) 0$

Here we assume $x \leftarrow e_{1} ; e_{2}$ as a shorthand for $\left(\lambda x . e_{2}\right) e_{1}$, and $e_{1} ; e_{2}$ for $\left(-\leftarrow e_{1} ; e_{2}\right)$. The evaluation of an operation clause now always returns directly with a function that takes the current state as its input; which is then used to resume with:

(handler $h\left(\lambda_{-}\right.$. perform set $21 ; x \leftarrow$ perform get ()$\left.\left.;(\lambda y \cdot x+x)\right)\right) 0$

$\longmapsto^{*}(\square 0) \cdot$ handle $h \cdot(\square ; x \leftarrow$ perform get ()$;(\lambda y \cdot x+x)) \cdot$ perform set 21

$\longmapsto^{*}(\square 0) \cdot(\lambda y \cdot k() 21) \quad$ with $k=\lambda x$. handle $h \cdot(\square ; x \leftarrow$ perform get ()$;(\lambda y \cdot x+x)) \cdot x$

$=(\lambda y \cdot k() 21) 0$

$\longmapsto k() 21$

$\longmapsto$ (handle $h \cdot(\square ; x \leftarrow$ perform get ()$;(\lambda y \cdot x+x)) \cdot()) 21$

$=(\square 21) \cdot$ handle $h \cdot(() ; x \leftarrow$ perform get ()$;(\lambda y \cdot x+x))$

$\longmapsto^{*} 42$ 
Clearly, defining local state as a function is quite cumbersome, so usually one allows for parameterized handlers [Bauer and Pretnar 2015a; Leijen 2017c; Pretnar 2010] that keep a local parameter $p$ with their handle frame, where the evaluation rules become:

phandler $h v^{\prime} v \quad \longrightarrow$ phandle $h v^{\prime} \cdot v()$

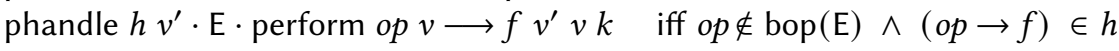

where $k=\lambda y x$. (phandle $h y \cdot \mathrm{E} \cdot x$ ). Here the handler parameter $v^{\prime}$ is passed to the operation clause $f$ and later restored in the resumption which now takes a fresh parameter $y$ besides the result value $x$. With a parameterized handler the state effect can be concisely defined as (variant 2):

$h=\{$ get $\rightarrow \lambda y x k . k y y$, set $\rightarrow \lambda y x k . k x()\}$

phandler $h 0\left(\lambda_{-}\right.$. perform set $21 ; x \leftarrow$ perform get ()$\left.; x+x\right)$

Another important advantage in this implementation is that the state effect is now tail resumptive which is beneficial for performance (as shown in the introduction).

Finally, there is another elegant way to implement local state by Biernacki et al. [2017], who define get and set operations in separate handlers (variant 3):

$$
\begin{aligned}
& h_{1}=\left\{\text { get } \rightarrow \lambda_{-} k . k 0\right\} \\
& h_{2}=\left\{\text { set } \rightarrow \lambda x k . \text { handler }\left\{\text { get } \rightarrow \lambda_{-} k . k x\right\}\left(\lambda_{-} . k()\right)\right\} \\
& \text { handler } h_{1}\left(\lambda_{-} \text {. handler } h_{2}\left(\lambda_{-} \text {. perform set } 42 ; x \leftarrow \text { perform get }() ; x+x\right)\right)
\end{aligned}
$$

Every set operation installs a fresh handler for the get operation and resumes under that (so the innermost get handler always contains the latest state). Even though elegant, there are some drawbacks to this encoding: a naïve implementation may use $n$ handler frames for $n$ set operations, typing this example is tricky and usually requires masking [Biernacki et al. 2017; Hillerström and Lindley 2016], and, as we will see, it does not use scoped resumptions and thus cannot be used with evidence translation.

Backtracking: By resuming more than once, we can implement backtracking using algebraic effects. For example, the $a m b$ effect handler collects all possible results in a list by resuming the flip operation first with true as result, and later again with false as result

$$
\begin{aligned}
& \text { handler }\left\{\text { flip } \rightarrow \lambda_{-} k . x s \leftarrow k \text { true; } y s \leftarrow k \text { false; } x s++y s\right\} \\
& \quad\left(\lambda_{-} . x \leftarrow \text { perform flip }() ; y \leftarrow \text { perform flip }() ;[x \& \& y]\right)
\end{aligned}
$$

returning the list [true, false, false, false] in our example. A similar technique can also be used to express probabilistic programming [Kiselyov and Shan 2009] with effect handlers.

Async: We can use resumptions $k$ as first-class values and for example store them into a queue to implement cooperative threads [Dolan et al. 2017] or asynchronous I/O [Leijen 2017b]. Assuming we have a state handler $h_{\text {queue }}$ that maintains a queue of pending resumptions, we can implement a scheduler as:

$$
\begin{aligned}
h_{\text {async }}=\left\{\begin{array}{l}
\text { fork } \rightarrow \\
\text { yield } \rightarrow
\end{array} \rightarrow \lambda_{-} k . \text { perform enqueue } k ; \text { schedule } f ; \text { next }()\right. \\
\text { next }=\lambda_{-} . k \leftarrow \text { perform dequeue }() ; k()
\end{aligned}
$$

Here, we assume enqueue enqueues a resumption $k$, and dequeue () returns one, or returns an identity function if the queue is empty. The schedule function runs a new action $f$ under the async handler:

$$
\begin{aligned}
& \text { schedule }=\lambda f . \text { handler } h_{\text {async }}\left(\lambda_{-} \cdot f()\right) \\
& \text { async }=\lambda f . \text { handler } h_{\text {queue }}\left(\lambda_{-} . \text {schedule } f\right)
\end{aligned}
$$

The main wrapper async schedules an action under a fresh scheduler queue handler $h_{\text {queue }}$, which is shared by all forked actions under it. 


\subsection{Scoped Resumptions}

The ability of effect handlers to capture the resumption as a first-class value is very powerful and can be considered as perhaps too powerful. In particular, it can be (ab)used to define handlers that change the semantics of other handlers that were defined and instantiated orthogonally. Take for example an operation $o p_{1}$ that is expected to always return the same result, say 1 . We can now define another operation $o p_{\text {evil }}$ that changes the return value of $o p_{1}$ after it is invoked! Consider the following program where we leave $f$ and $h_{\text {evil }}$ undefined for now:

$$
\begin{aligned}
& h_{1}=\left\{o p_{1} \rightarrow \lambda x k . k 1\right\} \\
& e=\text { perform } o p_{1}() ; \text { perform } o p_{\text {evil }}() \text {; perform } o p_{1}() \\
& \left.f \text { (handler } h_{1}\left(\lambda_{-} \text {. handler } h_{\text {evil }}\left(\lambda_{-} . e\right)\right)\right)
\end{aligned}
$$

Even though $h_{1}$ is defined as a pure reader effect and defined orthogonal to any other effect, the $o p_{\text {evil }}$ operation can still cause the second invocation of $o p_{1}$ to return 2 instead of 1 ! In particular, we can define $f$ and $h_{\text {evil }}$ as ${ }^{1}$ :

$$
\begin{aligned}
& h_{\text {evil }}=\left\{o p_{\text {evil }} \rightarrow \lambda \times k . k\right\} \\
& h_{2}=\left\{o p_{1} \rightarrow \lambda x k . k 2\right\} \\
& f=\lambda k . \text { handler } h_{2}\left(\lambda_{-} . k()\right)
\end{aligned}
$$

The trick is that the handler $h_{\text {evil }}$ does not directly resume but instead returns the resumption $k$ as $i s$, after unwinding through $h_{1}$ it is passed to $f$ which now invokes the resumption $k$ under a fresh handler $h_{2}$ for $o p_{1}$ causing all subsequent $o p_{1}$ operations to be handled by $h_{2}$ instead.

We consider this behavior undesirable in practice as it limits the ability to do local reasoning. In particular, a programmer may not expect that calling $o p_{\text {evil }}$ changes the semantics of $o p_{1}$. Yet there is no way to forbid it. Moreover, it also affects static analysis and it turns out for example that efficient evidence translation (with its subsequent performance benefits) is not possible if we allow resumptions to be this dynamic.

The solution we propose in this paper is to limit resumptions to be scoped only: that is, $a$ resumption can only be applied under the same handler context as it was captured. The handler context is the evaluation context where we just consider the handler frames, e.g. for any evaluation context $\mathrm{E}$ of the form $\mathrm{F}_{0} \cdot$ handle $h_{1} \cdot \mathrm{F}_{1} \cdot \ldots \cdot$ handle $h_{n} \cdot \mathrm{F}_{n}$, the handler context, $\operatorname{hctx}(\mathrm{E})$, is $h_{1} \cdot h_{2} \cdot \ldots \cdot h_{n}$. In particular, the evil example is rejected as it does not use a scoped resumption: $k$ is captured under $h_{1}$ but applied under $h_{2}$.

Our definition of scoped resumption is minimal in the sense that it is the minimal requirement needed in the proofs to maintain coherence of evidence translation. In this paper, we guarantee scoped resumptions using a dynamic runtime check in evidence translated programs (called guard), but it is also possible to check it statically. It is beyond the scope of this paper to give a particular design, but some ways of doing this are:

- Lexical scoping: a straightforward approach is to syntactically restrict the use of the resumption to be always in the lexical scope of the handler: i.e. fully applied within the operation clause and no occurrences under a lambda (so it cannot escape or be applied in nested handler). This can perhaps already cover all reasonable effects in practice, especially in combination with parameterized handlers ${ }^{2}$.

- A more sophisticated solution could use generative types for handler names, together with a check that those types do not escape the lexical scope as described by Zhang and Myers [2019] and also used by Biernacki et al. [2019] and Brachthäuser et al. [2020]. Another option could

\footnotetext{
${ }^{1}$ Note that this example is fine in $\lambda^{\epsilon}$ but cannot be typed in $\mathrm{F}^{\epsilon}$ as is - we discuss a properly typed version in Section 4.5. ${ }^{2}$ The lexical approach could potentially be combined with an "unsafe" resumption that uses a runtime check as done in this article to cover any remaining situations.
} 
be to use rank-2 types to prevent the resumption from escaping the lexical scope in which the handler is defined [Leijen 2014; Peyton Jones and Launchbury 1995].

In the seminal work on algebraic effect handlers by Plotkin and Pretnar [2013] the resumptions $k$ are in a separate syntactic class, always fully applied, and checked under a context $K$ separate from $\Gamma$. However, they still allow occurrences under a lambda, allowing a resumption to escape. If we would adapt just the lambda rule to check the premise under an empty environment $K$, then all resumptions are scoped (implementing the lexical scoping rule).

\subsection{Expressiveness}

Scoped resumptions bring easier-to-reason control flow, and, as we will see, open up new design space for algebraic effects compilation. However, one might worry about the expressiveness of scoped resumptions. We believe that all important effect handlers in practice can be defined in terms of scoped resumptions. In particular, note that it is still allowed for a handler to grow its context with applicative forms, for example:

handler $\{$ tick $\rightarrow \lambda x k .1+k()\}\left(\lambda_{-}\right.$. perform tick (); perform tick (); 1)

evaluates to 3 by keeping $(1+\square)$ frames above the resumption. In this example, even though the full context has grown, $k$ is still a scoped resumption as it resumes under the same (empty) handler context. Similarly, the async scheduler example that stores resumptions in a stateful queue is also accepted since each resumption is applied under the same handler context (with the state queue handler on top). Multiple resumptions as in the backtracking example are also admitted.

We identify three classes of programs that cannot be expressed with scoped resumptions. First, the state variant 3 based on two separate handlers does not use scoped resumptions since the set resumption resumes always under a handler context extended with a get handler. However, we can always use, and due to the reasons we have mentioned we may actually prefer, the normal state effect or the parameterized state effect. Second, shallow handlers do not resume under their own handler and as a result generally resume under a different handler context than they captured. Fortunately, any program with shallow handler can be expressed with deep handlers as well [Hillerström and Lindley 2018; Kammar et al. 2013] and thus avoid the unscoped resumptions. Finally, Kiselyov et al. [2006] show an example of code migration that resumes locally captured continuations on another host, possibly under different handlers.

\section{EXPLICITLY TYPED EFFECT HANDLERS IN SYSTEM $\mathrm{F}^{\epsilon}$}

To prepare for a type directed evidence translation, we first define a typed version of the untyped calculus $\lambda^{\epsilon}$ called System $\mathrm{F}^{\epsilon}$ - a call-by-value effect handler calculus extended with (higher-rank impredicative) polymorphic types and higher kinds à la System $\mathrm{F}_{\omega}$, and row based effect types. Figure 2 defines the extended syntax and evaluation rules with the syntax of types and kinds in Figure 3. System $\mathrm{F}^{\epsilon}$ serves as an explicitly typed calculus that can be the target language of compilers and, for this article, serves as the basis for type directed evidence translation.

Being explicitly typed, we now have type applications $e[\sigma]$ and abstractions $\Lambda \alpha^{k}$. $v$. Also, $\lambda^{\epsilon} x: \sigma . e$, handle ${ }^{\epsilon} h e$, handler ${ }^{\epsilon} h$, and perform ${ }^{\epsilon} o p \bar{\sigma}$ all carry an effect type $\epsilon$. Effect types are (extensible) rows of effect labels $l$ (like exn or state). In the types, every function arrow $\sigma_{1} \rightarrow \epsilon \sigma_{2}$ takes three arguments: the input type $\sigma_{1}$, the output type $\sigma_{2}$, and its effects $\epsilon$ when it is evaluated. When $\epsilon$ is an empty row, we often omit the effect annotation.

Since we have effect rows, effect labels, and regular value types, we use a basic kind system to keep them apart and to ensure well-formedness ( $\left.t_{\mathrm{wf}}\right)$ of types (as defined in the technical report [Xie et al. 2020]). 


\begin{tabular}{|c|c|c|c|c|c|}
\hline Expre & sions & & Values & & \\
\hline$e::=$ & $v$ & (value) & $v::=$ & $x$ & (variables) \\
\hline & & (application) & | & $\lambda^{\epsilon} x: \sigma . e$ & (abstraction) \\
\hline & $e[\sigma]$ & (type application) & & $\Lambda \alpha^{k} \cdot v$ & (type abstraction) \\
\hline & handle $h e$ & (handler instance) & & $\begin{array}{l}\text { handler }{ }^{\epsilon} h \\
\text { perform }^{\epsilon} \text { op } \bar{\sigma}\end{array}$ & $\begin{array}{l}\text { (effect handler) } \\
\text { (operation) }\end{array}$ \\
\hline
\end{tabular}
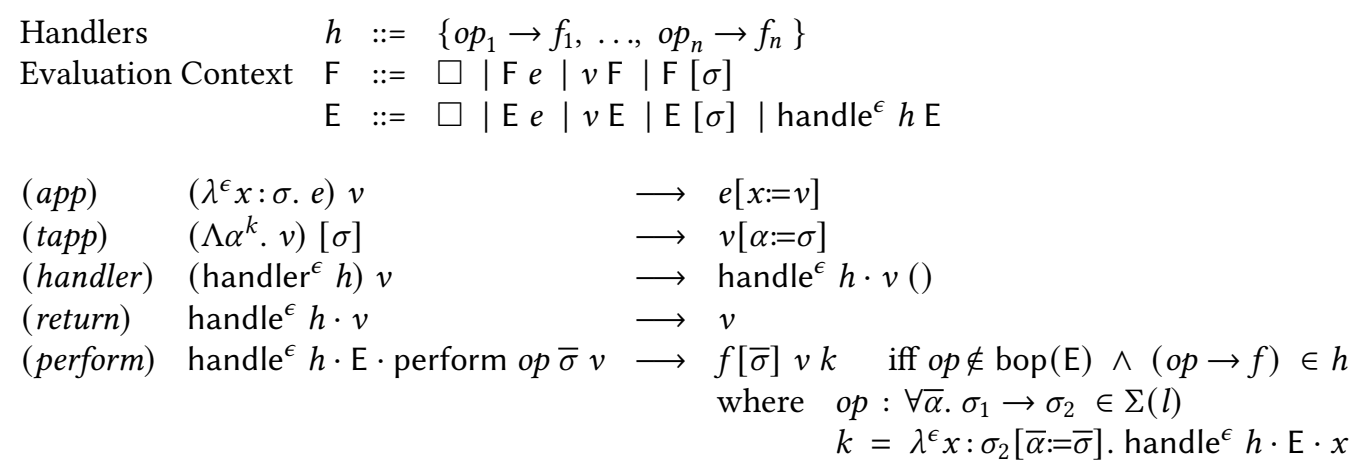

Fig. 2. System $\mathrm{F}^{\epsilon}$ : explicitly typed algebraic effect handlers. Figure 3 defines the types.

\begin{tabular}{|c|c|c|c|c|c|}
\hline Types & & & Kinds & & \\
\hline$\sigma::=$ & $\alpha^{k}$ & (type variables of kind $k$ ) & $k::=$ & $*$ & (value type) \\
\hline & $c^{k} \sigma \ldots \sigma$ & (type constructor of kind $k$ ) & & $k \rightarrow k$ & (type constructors) \\
\hline & $\sigma \rightarrow \epsilon \sigma$ & (function type) & & eff & (effect type $(\mu, \epsilon))$ \\
\hline & $\forall \alpha^{k} \cdot \sigma$ & (quantified type) & & lab & (basic effect $(l))$ \\
\hline
\end{tabular}
Effect signature sig $\quad::=\left\{o p_{1}: \forall \bar{\alpha}_{1}, \sigma_{1} \rightarrow \sigma_{1}^{\prime}, \ldots, o p_{n}: \forall \bar{\alpha}_{n} . \sigma_{n} \rightarrow \sigma_{n}^{\prime}\right\}$
Effect signatures $\quad \Sigma \quad::=\left\{l_{1}: \operatorname{sig}_{1}, \ldots, l_{n}: \operatorname{sig}_{n}\right\}$
Type Constructors \langle\rangle$\quad:$ eff empty effect row (total)
\langle|$\_\quad: \quad$ lab $\rightarrow$ eff $\rightarrow$ eff $\quad$ effect row extension

Syntax $\quad\left\langle l_{1}, \ldots, l_{n}\right\rangle \doteq\left\langle l_{1}|\ldots|\left\langle l_{n} \mid\langle\rangle\right\rangle \ldots\right\rangle$

$\left\langle l_{1}, \ldots, l_{n} \mid \mu\right\rangle \doteq\left\langle l_{1}|\ldots|\left\langle l_{n} \mid \mu\right\rangle \ldots\right\rangle$

$\epsilon::=\sigma^{\text {eff }}, \quad \mu::=\alpha^{\text {eff }}, \quad l::=c^{\text {lab }}$

Fig. 3. System $F^{\epsilon}$ : types

$$
\begin{array}{cc}
\frac{}{\epsilon \equiv \epsilon}[\mathrm{REFL}] & \frac{\epsilon_{1} \equiv \epsilon_{2}}{\epsilon_{1} \equiv \epsilon_{3}} \text { [EQ-TRANS] } \\
\frac{l_{1} \neq l_{2}}{\epsilon_{1} \equiv \epsilon_{2}} & \frac{\epsilon_{1} \equiv \epsilon_{2}}{\left\langle l \mid \epsilon_{1}\right\rangle \equiv\left\langle l \mid \epsilon_{2}\right\rangle} \text { [EQ-HEAD] }
\end{array}
$$

Fig. 4. Equivalence of row-types. 


\subsection{Effect Rows}

An effect row is either empty \langle\rangle (the total effect), a type variable $\mu$ (of kind eff), or an extension $\langle l \mid \epsilon\rangle$ where $\epsilon$ is extended with effect label $l$. We call effects that end in an empty effect closed, i.e. $\left\langle l_{1}, \ldots, l_{n}\right\rangle$; and effects that end in a polymorphic tail open, i.e. $\left\langle l_{1}, \ldots, l_{n} \mid \mu\right\rangle$. Following Leijen [2014] and Biernacki et al. [2017], we use simple effect rows where labels can be duplicated, and where an effect $\langle l, l\rangle$ is not equal to $\langle l\rangle$. We consider rows equivalent up to the order of the labels as defined in Figure 4. Note rule EQ-swAP only swaps distinct labels. Following Leijen [2014], we disallow polymorphism over labels. There exists a complete and sound unification algorithm for these row types [Leijen 2005] and thus these are also very suitable for Hindley-Milner style type inference.

We consider using simple row-types with duplicate labels a suitable choice for a core calculus since it extends System F typing seamlessly as we only extend the notion of equality between types. There are other approaches to typing effects but all existing approaches depart from standard System $\mathrm{F}$ typing in significant ways. Row typing without duplicate labels leads to the introduction of type constraints, as in T-REX for example [Gaster and Jones 1996], or kinds with presence variables (Rémy style rows) as in Links for example [Hillerström and Lindley 2016; Rémy 1994] Another approach is using effect subtyping [Bauer and Pretnar 2014] but that requires a subtype relation between types instead of simple equality.

The reason we need equivalence between row types up to order of effect labels is due to polymorphism. Suppose we have two functions that each use different effects:

$$
f_{1}: \forall \mu .() \rightarrow\left\langle l_{1} \mid \mu\right\rangle() \quad f_{2}: \forall \mu .() \rightarrow\left\langle l_{2} \mid \mu\right\rangle()
$$

We would still like to be able to express choose $f_{1} f_{2}$ where choose $\forall \alpha . \alpha \rightarrow \alpha \rightarrow \alpha$. Using row types we can type this naturally as:

$$
\Lambda \mu \text {. choose }\left[() \rightarrow\left\langle l_{1}, l_{2} \mid \mu\right\rangle()\right]\left(f_{1}\left[\left\langle l_{2} \mid \mu\right\rangle\right]\right)\left(f_{2}\left[\left\langle l_{1} \mid \mu\right\rangle\right]\right)
$$

where the types of the arguments are now equivalent $\left\langle l_{1} \mid\left\langle l_{2} \mid \mu\right\rangle\right\rangle \equiv\left\langle l_{2} \mid\left\langle l_{1} \mid \mu\right\rangle\right\rangle$ (without needing subtype constraints or polymorphic label flags).

Similarly, duplicate labels can easily arise due to type instantiation. For example, a catch handler for exceptions can have type:

$$
\text { catch }: \forall \mu \alpha .(() \rightarrow\langle\text { exn } \mid \mu\rangle \alpha) \rightarrow(\text { string } \rightarrow \mu \alpha) \rightarrow \mu \alpha
$$

where catch takes an action that can raise exceptions, and a handler function that is called when an exception is caught. Suppose though an exception handler itself raises an exception, and has type $h: \forall \mu$. string $\rightarrow\langle$ exn $\mid \mu\rangle$ int. The application catch action $h$ is then explicitly typed as:

$\Lambda \mu$. catch $[\langle\operatorname{exn} \mid \mu\rangle$, int $]$ action $h[\mu]$

where the type application gives rise to the type:

$$
\operatorname{catch}[\langle\operatorname{exn} \mid \mu\rangle \text {, int }]:(() \rightarrow\langle\text { exn, exn } \mid \mu\rangle \text { int }) \rightarrow(\text { string } \rightarrow\langle\text { exn } \mid \mu\rangle \text { int }) \rightarrow\langle\text { exn } \mid \mu\rangle \text { int }
$$

naturally leading to duplicate labels in the type. As we will see, simple row types also correspond naturally to the shape of the runtime evidence vectors that we introduce in Section 4.1 (where duplicated labels correspond to nested handlers)

\subsection{Operations}

We assume that every effect $l$ has a unique set of operations $o p_{1}$ to $o p_{n}$ with a signature sig that gives every operation its input and output types, $o p_{i}: \forall \bar{\alpha}_{i} . \sigma_{i} \rightarrow \sigma_{i}^{\prime}$. There is a global map $\Sigma$ that maps each effect $l$ to its signature. Since we assume that each $o p$ is uniquely named, we use the notation op $: \forall \bar{\alpha} . \sigma_{1} \rightarrow \sigma_{2} \in \Sigma(l)$ to denote the type of op that belongs to effect $l$, and also op $\in \Sigma(l)$ to signify that op is part of effect $l$. Moreover, we use the notation $h: \Sigma(l)$ to mean that $h$ corresponds to $l$ (for any op $\in h, o p \in \Sigma(l)$ ). 
Note that we allow operations to be polymorphic. Therefore perform op $\bar{\sigma} v$ contains the instantiation types $\bar{\sigma}$ which are passed to the operation clause $f$ in the evaluation rule for (perform) (Figure 2). This means that operations can be used polymorphically, but the handling clause itself must be polymorphic in the operation types (and use them as abstract types).

\subsection{Quantification and Equivalence to the Untyped Dynamic Semantics}

Erasing types from System $\mathrm{F}^{\epsilon}$ should not affect operational semantics, i.e.

Theorem 1. (System $\mathrm{F}^{\epsilon}$ has untyped dynamic semantics) If $e_{1} \longrightarrow e_{2}$ in System $\mathrm{F}^{\epsilon}$, then either $e_{1}^{*} \longrightarrow e_{2}^{*}$ or $e_{1}^{*}=e_{2}^{*}$.

where $e^{*}$ stands for the term $e$ with all types, type abstractions, and type applications removed. This seems an obvious property but there is a subtle interaction with quantification. Suppose we (wrongly) allow quantification over expressions instead of values, like $\Lambda \alpha$. $e$, then consider:

$$
h=\{\text { tick } \rightarrow \lambda x:() k:(() \rightarrow\langle\rangle \text { int }) .1+k()\}
$$

handle $h((\lambda x: \forall \alpha$. int. $x[$ int $]+x[$ bool $])(\Lambda \alpha$. tick ()$; 1))$

In the typed semantics, this would evaluate the argument $x$ at each instantiation (since the whole $\Lambda \alpha$. tick (); 1 is passed as a value), resulting in 4 . On the other hand, if we perform type erasure, the untyped dynamic semantics evaluates to 3 instead (evaluating the argument before applying). Not only do we lose untyped dynamic semantics, but we also break parametricity (as we can observe instantiations). So, it is quite important to only allow quantification over values, much like the ML value restriction [Kammar and Pretnar 2017; Pitts 1998; Wright 1995]. In the proof of Theorem 1 we use in particular the following (seemingly obvious) lemma:

Lemma 1. (Type erasure of values)

If $v$ is a value in System $\mathrm{F}^{\epsilon}$ then $v^{*}$ is a value in $\lambda^{\epsilon}$.

Not all systems in the literature adhere to this restriction; for example Biernacki et al. [2017] and Leijen [2017c] allow quantification over expressions as $\Lambda \alpha$. e, where both ensure soundness of the effect type system by disallowing type abstraction over effectful expressions. However, we believe that this remains a risky affair since Lemma 1 does not hold; and thus a typed evaluation may take more reduction steps than the type-erased term, i.e. seemingly shared argument values may be computed more than once.

\subsection{Type Rules for System F ${ }^{\epsilon}$}

Figure 5 defines the typing rules for System $\mathrm{F}^{\epsilon}$. The rules are of the form $\Gamma ; w \vdash e: \sigma \mid \epsilon \rightsquigarrow e^{\prime}$ for expressions where the variable context $\Gamma$ and the effect $\epsilon$ are inherited $(\uparrow)$, and $\sigma$ is synthesized $(\downarrow)$. The gray parts define the evidence translation, which we describe in Section 4, and can be ignored for now. Values are not effectful and are typed as $\Gamma \vdash_{\text {val }} \quad v: \sigma \rightsquigarrow v^{\prime}$. Since effects are inherited, lambda expressions need an effect annotation that is passed to the body derivation (ABs). In the rule APP we use standard equality between types and require that all effects match. The vAL rule goes from a value to an expression (opposite of ABs) and allows any inherited effect. The HANDLER rule takes an action with effect $\langle l \mid \epsilon\rangle$ and handles $l$ leaving effect $\epsilon$. The HANDLE rule is similar, but is defined over an expression $e$ and types $e$ under an extended effect $\langle l \mid e\rangle$ in the premise. In the rule ops, we implicitly assume $\left\{o p_{1}, \ldots, o p_{n}\right\}=\Sigma(l)$.

See the technical report [Xie et al. 2020] for the full type rules for evaluation contexts. The judgement $\Gamma \vdash_{\mathrm{ec}} \mathrm{E}: \sigma_{1} \rightarrow \sigma_{2} \mid \epsilon$ signifies that a context $\mathrm{E}$ can be typed as a function from a term of type $\sigma_{1}$ to $\sigma_{2}$ where the resulting expression has effect $\epsilon$. These rules are not needed to check programs but are very useful in proofs and theorems. In particular, 


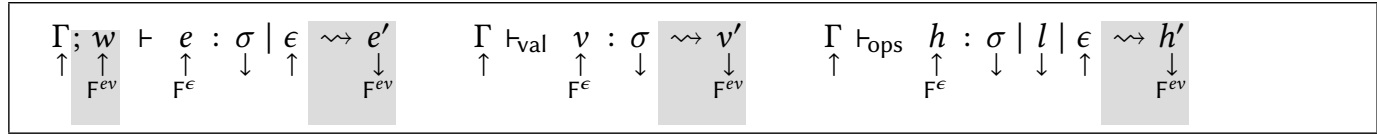

$$
\begin{aligned}
& \frac{x: \sigma \in \Gamma}{\Gamma \vdash_{\mathrm{val}} x: \sigma \rightsquigarrow x}[\mathrm{VAR}] \frac{\Gamma, x: \sigma_{1} ; z \vdash e: \sigma_{2} \mid \epsilon \rightsquigarrow e^{\prime} \quad \text { fresh } z}{\Gamma \vdash_{\mathrm{val}} \lambda^{\epsilon} x: \sigma_{1} . e: \sigma_{1} \rightarrow^{\epsilon} \sigma_{2} \rightsquigarrow \lambda^{\epsilon} z: \operatorname{evv} \epsilon, x:\left\lceil\sigma_{1}\right\rceil . e^{\prime}}[\mathrm{ABS}] \\
& \frac{\Gamma \vdash_{\mathrm{val}} v: \sigma \rightsquigarrow v^{\prime}}{\Gamma ; w \vdash v: \sigma \mid \epsilon \rightsquigarrow v^{\prime}}[\mathrm{vAL}] \quad \frac{\Gamma \vdash_{\mathrm{val}} v: \sigma \rightsquigarrow v^{\prime} \quad k \neq \mathrm{lab}}{\Gamma \vdash_{\mathrm{val}} \Lambda \alpha^{k} . v: \forall \alpha^{k} . \sigma \rightsquigarrow \Lambda \alpha^{k} . v^{\prime}} \text { [TABs] } \\
& \Gamma ; w \vdash e_{1}: \sigma_{1} \rightarrow \epsilon \sigma \mid \epsilon \rightsquigarrow e_{1}^{\prime} \\
& \frac{\Gamma ; w \vdash e_{2}: \sigma_{1} \mid \epsilon \rightsquigarrow e_{2}^{\prime}}{\Gamma ; w \vdash e_{1} e_{2}: \sigma \mid \epsilon \rightsquigarrow e_{1}^{\prime} w e_{2}^{\prime}}[\mathrm{APP}] \frac{\Gamma ; w \vdash e: \forall \alpha^{k} . \sigma_{1} \mid \epsilon \rightsquigarrow e^{\prime} \vdash_{\mathrm{wf}} \sigma: k}{\left.\Gamma ; w \vdash e[\sigma]: \sigma_{1}[\alpha:=\sigma] \mid \epsilon \rightsquigarrow e^{\prime}[\sigma \sigma]\right]} \text { [TAPP] } \\
& \frac{o p: \forall \bar{\alpha} . \sigma_{1} \rightarrow \sigma_{2} \in \sum(l) \quad \bar{\alpha} \notin \mathrm{ftv}(\Gamma)}{\Gamma \vdash_{\text {val }} \text { perform }^{\epsilon} \text { op } \bar{\sigma}: \sigma_{1}[\bar{\alpha}:=\bar{\sigma}] \rightarrow\langle l \mid \epsilon\rangle \sigma_{2}[\bar{\alpha}:=\bar{\sigma}] \rightsquigarrow \text { perform }^{\epsilon} \text { op } \bar{\sigma}} \text { [PERFORM] } \\
& o p_{i}: \forall \bar{\alpha} . \sigma_{1} \rightarrow \sigma_{2} \in \Sigma(l) \quad \bar{\alpha} \pitchfork \mathrm{ftv}(\epsilon \sigma) \\
& \frac{\Gamma r_{\text {val }} f_{i}: \forall \bar{\alpha} . \sigma_{1} \rightarrow \epsilon\left(\left(\sigma_{2} \rightarrow \epsilon \sigma\right) \rightarrow \epsilon \sigma\right) \rightsquigarrow f_{i}^{\prime}}{\Gamma \text { rops }_{\text {o }}\left\{o p_{1} \rightarrow f_{1}, \ldots, o p_{n} \rightarrow f_{n}\right\}: \sigma|l| \epsilon \rightsquigarrow\left\{o p_{i} \rightarrow f_{i}^{\prime}\right\}} \text { [ops] } \\
& \frac{\Gamma \vdash_{\text {ops }} h: \sigma|l| \epsilon \rightsquigarrow h^{\prime}}{\Gamma \vdash_{\text {val }} \text { handler }^{\epsilon} h:(() \rightarrow\langle l \mid \epsilon\rangle \sigma) \rightarrow \epsilon \sigma \rightsquigarrow \text { handler }^{\epsilon} h^{\prime}} \text { [HANDLER] } \\
& \frac{\Gamma \vdash_{\mathrm{ops}} h: \sigma|l| \epsilon \rightsquigarrow h^{\prime} \quad \Gamma ;\left\langle l:\left(m, h^{\prime}\right) \mid w\right\rangle \vdash e: \sigma \mid\langle l \mid \epsilon\rangle \rightsquigarrow e^{\prime} \quad m \text { fresh }}{\Gamma ; w \vdash \text { handle }^{\epsilon} h e: \sigma \mid \epsilon \rightsquigarrow \text { handle }_{m}^{w} h^{\prime} e^{\prime}} \text { [HANDLE] }
\end{aligned}
$$

Fig. 5. Type Rules for System $\mathrm{F}^{\epsilon}$ combined with type directed evidence translation to $\mathrm{F}^{e v}$ (in gray.)

Lemma 2. (Evaluation context typing)

If $\varnothing \vdash_{\text {ec }} \mathrm{E}: \sigma_{1} \rightarrow \sigma \mid \epsilon$ and $\varnothing \vdash e: \sigma_{1} \mid\left\langle[\mathrm{E}\rceil^{l} \mid \epsilon\right\rangle$, then $\varnothing \vdash \mathrm{E}[e]: \sigma \mid \epsilon$.

where $\lceil\mathrm{E}\rceil^{l}$ extracts all labels $l$ from a context in reverse order:

$\left\lceil\mathrm{F}_{0} \cdot \text { handle } h_{1} \cdot \mathrm{F}_{1} \cdot \ldots \text { handle } h_{n} \cdot \mathrm{F}_{n}\right\rceil^{l}=\left\langle l_{n}, \ldots, l_{1}\right\rangle$ iff $h_{i}: \Sigma\left(l_{i}\right)$

The above lemma shows we can plug well-typed expressions in a suitable context. The next lemma uses this to show the correspondence between the dynamic evaluation context and the static effect type:

Lemma 3. (Effect corresponds to the evaluation context)

If $\varnothing \vdash \mathrm{E}[e]: \sigma \mid \epsilon$ then there exists $\sigma_{1}$ such that $\varnothing \vdash_{\mathrm{ec}} \mathrm{E}: \sigma_{1} \rightarrow \sigma \mid \epsilon$, and $\varnothing \vdash e: \sigma_{1} \mid\left\langle\lceil\mathrm{E}\rceil^{l} \mid \epsilon\right\rangle$.

Here we see that the rules guarantee that exactly the effects $\lceil\mathrm{E}\rceil^{l}$ in $e$ are handled by the context $\mathrm{E}$.

\subsection{Progress and Preservation}

We establish two essential lemmas about the meaning of effect types. First, in any well-typed total System $\mathrm{F}^{\epsilon}$ expression, all operations are handled (and thus, evaluation cannot get stuck): 
Lemma 4. (Well typed operations are handled)

If $\varnothing \vdash \mathrm{E}[$ perform op $\bar{\sigma} v]: \sigma \mid\langle\rangle$ then $\mathrm{E}$ has the form $\mathrm{E}_{1} \cdot$ handle $^{\epsilon} h \cdot \mathrm{E}_{2}$ with $o p \notin \operatorname{bop}\left(\mathrm{E}_{2}\right)$ and $o p \rightarrow f \in h$.

Moreover, effect types are meaningful in the sense that an effect type fully reflects all possible effects that may happen during evaluation:

Lemma 5. (Effects types are meaningful)

If $\varnothing \vdash \mathrm{E}[$ perform op $\bar{\sigma} v]: \sigma \mid \epsilon$ with $o p \notin \operatorname{bop}(\mathrm{E})$, then $o p \in \Sigma(l)$ and $l \in \epsilon$, i.e. effect types cannot be discarded without a handler.

Using these lemmas, we can show that evaluation can always make progress and that the typings are preserved during evaluation.

Theorem 2. (Progress)

If $\varnothing \vdash e_{1}: \sigma \mid\langle\rangle$ then either $e_{1}$ is a value, or $e_{1} \longmapsto e_{2}$.

Theorem 3. (Preservation)

If $\varnothing \vdash e_{1}: \sigma \mid\langle\rangle$ and $e_{1} \longmapsto e_{2}$, then $\varnothing \vdash e_{2}: \sigma \mid\langle\rangle$.

\section{POLYMORPHIC EVIDENCE TRANSLATION TO SYSTEM F ${ }^{e v}$}

Having established a sound explicitly typed core calculus, we are ready to present evidence translation. The goal of evidence translation is to pass handler implementations as part of an evidence vector. The handler implementation is passed from the point where the handler was introduced to the point where the effect operation is performed. Passing evidence explicitly will in turn enable other optimizations (as described in Section 6) since we can now locally inspect the evidence instead of searching in the dynamic evaluation context.

Following Brachthäuser and Schuster [2017], we represent evidence $e v$ for an effect $l$ as a pair $(m, h)$, consisting of a unique marker $m$ and the corresponding handler implementation $h$. The markers uniquely identify each handler frame in the context which is now marked as handle ${ }_{m} h$. The reason for introducing the separate handler $h$ construct is now apparent: it instantiates handle $m$ frames with a unique $m$. This representation of evidence allows for two important optimizations: (1) We can change the operational rule for perform to directly yield to a particular handler identified by $m$ (instead of needing to search for the innermost one), shown in Section 5.1, and (2) It allows local inspection of the actual handler $h$ so we can evaluate tail resumptive operations in place, shown in Section 6.

However, passing the evidence to each operation turns out to be surprisingly tricky to get right and we took quite a few detours before arriving at the current solution. At first, we thought we could represent evidence for each label $l$ in the effect of a function as separate argument $e v l$. For example,

$$
f_{1}: \forall \mu \text {. int } \rightarrow\left\langle l_{1} \mid \mu\right\rangle \text { int }=\Lambda \mu . \lambda x \text {. perform } o p_{1} x
$$

would be translated as:

$$
f_{1}: \forall \mu \text {. ev } l_{1} \rightarrow \text { int } \rightarrow\left\langle l_{1} \mid \mu\right\rangle \text { int }=\Lambda \mu \text {. } \lambda e v . \lambda x \text {. perform op } p_{1} \text { ev } x
$$

This does not work though as type instantiation can now cause the runtime representation to change as well! For example, if we instantiate $\mu$ to $\left\langle l_{2}\right\rangle$ as $f_{1}\left[\left\langle l_{2}\right\rangle\right]$ the type becomes int $\rightarrow\left\langle l_{1}, l_{2}\right\rangle$ int which now takes two evidence parameters. Even worse, such instantiation can be inside arbitrary types, like a list of such functions, where we cannot construct evidence transformers in general.

Another design that does not quite work is to regard evidence translation as an instance of qualified types [Jones 1992] and use a dictionary passing translation. In essence, in the theory of 


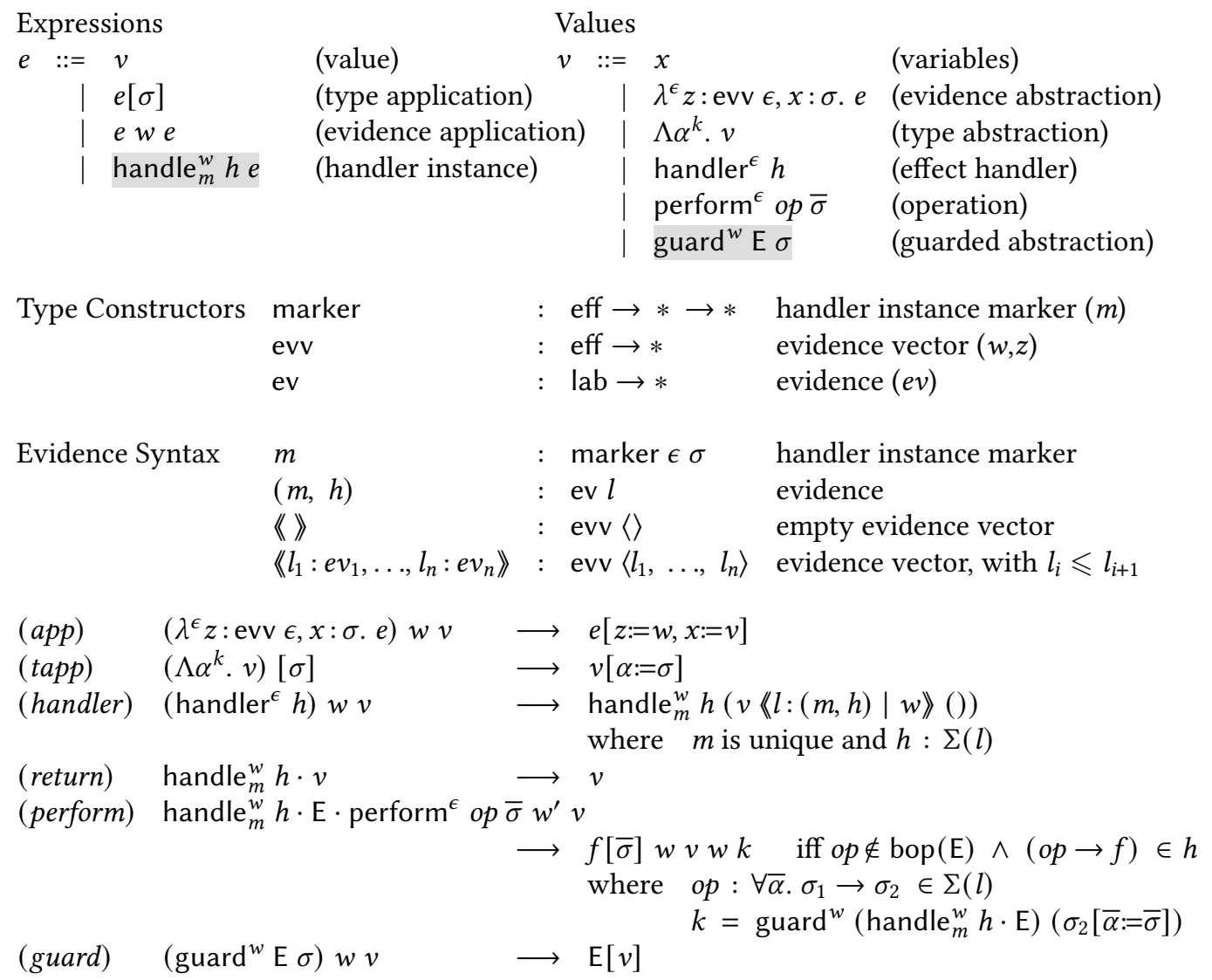

Fig. 6. System $\mathrm{F}^{e v}$ Typed operational semantics with evidence

qualified types, the qualifiers are scoped over monomorphic types, which does not fit well with effect handlers. Suppose we have a function foo with a qualified evidence types as:

foo $: E v l_{1} \Rightarrow\left(\right.$ int $\rightarrow\left\langle l_{1}\right\rangle$ int $) \rightarrow\left\langle l_{1}\right\rangle$ int

Note that even though foo is itself qualified, the argument it takes is a plain function int $\rightarrow\left\langle l_{1}\right\rangle$ int and has already resolved its own qualifiers. That is too eager for our purposes. For example, if we apply foo $\left(f_{1}[\langle\rangle]\right)$, under dictionary translation we would get foo e $v_{1}\left(f_{1}[\langle\rangle] e v_{1}\right)$. However, it may well be that foo itself applies $f_{1}$ under a new handler for the $l_{1}$ effect and thus needs to pass different evidence than $e v_{1}$ ! Effectively, dictionary translation may partially apply functions with their dictionaries which is not legal for handler evidence. The qualified type we really require for foo uses higher-ranked qualifiers, something like $E v l_{1} \Rightarrow\left(E v l_{1} \Rightarrow\right.$ int $\rightarrow\left\langle l_{1}\right\rangle$ int $) \rightarrow\left\langle l_{1}\right\rangle$ int.

\subsection{Evidence Vectors}

The design we present here instead passes all evidence as a single evidence vector to each (effectful) function: this keeps the runtime representations stable under type instantiation, and we can ensure syntactically that functions are never partially applied to evidence. 
Figure 6 defines our target language $\mathrm{F}^{e v}$ as an explicitly typed calculus with evidence passing. All applications are now of the form $e_{1} w e_{2}$ where we always pass an evidence vector $w$ with the original argument $e_{2}$. Therefore, all lambdas are of the form $\lambda^{\epsilon} z: \operatorname{evv} \epsilon, x: \sigma . e$ and always take an evidence vector $z$ besides their regular parameter $x$. Also, handle ${ }_{m}$ frames now carry the evidences and become handle ${ }_{m}^{w}$. We also extend application forms in the evaluation context to take evidence parameters. The double arrow notation is used do denote the type of these "tupled" lambdas:

$$
\sigma_{1} \Rightarrow \epsilon \sigma_{2} \doteq \operatorname{evv} \epsilon \rightarrow \sigma_{1} \rightarrow \epsilon \sigma_{2}
$$

During evidence translation, every effect type $\epsilon$ on an arrow is translated to an explicit runtime evidence vector of type evv $\epsilon$, and we translate type annotations as:

$$
\begin{array}{ll}
\lceil\cdot\rceil: k \rightarrow k & \\
\lceil\forall \alpha . \sigma\rceil=\forall \alpha .\lceil\sigma\rceil & \lceil\alpha\rceil \quad=\alpha \\
\left\lceil\tau_{1} \rightarrow \epsilon \tau_{2}\right\rceil=\left\lceil\tau_{1}\right\rceil \Rightarrow \epsilon\left\lceil\tau_{2}\right\rceil & \left\lceil c \tau_{1} \ldots \tau_{n}\right\rceil=c\left\lceil\tau_{1}\right\rceil \ldots\left\lceil\tau_{n}\right\rceil
\end{array}
$$

Evidence vectors $\left\langle l_{1}: e v_{1}, \ldots, l_{n}: e v_{n}\right\rangle$ are essentially a map from effect labels to evidence. Later we want to be able to select evidence from a vector with a constant offset instead of searching for the label, so we are going to keep them in a canonical form ordered by the effect types $l$. That is, we have every $l_{i} \leqslant l_{i+1}$. We also use the notation $\langle l: e v, w\rangle$ to decompose an evidence vector into a head label $l$ with evidence $e v$ and a tail evidence vector $w$ (maintaining canonical forms). An empty evidence vector is denoted as $《 \gg$.

During evaluation we need to be able to select evidence from an evidence vector, and to insert new evidence when a handler is instantiated, and we define the following two operations:

$$
\begin{array}{lll}
. l & : \forall \mu . \operatorname{evv}\langle l \mid \mu\rangle \rightarrow \operatorname{ev} l & \text { (select evidence from a vector) } \\
\left\langle l:_{-} \mid{ }_{-}\right\rangle & : \forall \mu . \operatorname{ev} l \rightarrow \operatorname{evv} \mu \rightarrow \operatorname{evv}\langle l \mid \mu\rangle & \text { (evidence insertion) }
\end{array}
$$

Where we assume the following two laws that relate selection and insertion:

$$
\begin{aligned}
& \langle l: e v \mid w\rangle . l=e v \\
& \left\langle l^{\prime}: e v \mid w\right\rangle . l=w . l \quad \text { iff } l \neq l^{\prime}
\end{aligned}
$$

The evidence insertion operation inserts an evidence into an evidence vector in an ordered way:

$$
\begin{aligned}
& \left\langle l:_{-} \mid \_\right\rangle: \forall \mu \text {. ev } l \rightarrow \operatorname{evv} \mu \rightarrow \operatorname{evv}\langle l \mid \mu\rangle \\
& \langle l: e v \mid\langle\rangle\rangle\rangle=\langle l: e v\rangle \\
& \left.\left\langle l: e v \mid\left\langle l^{\prime}: e v^{\prime}, w\right\rangle\right\rangle=\left\langle l^{\prime}: e v^{\prime},\langle l: e v \mid w\rangle\right\rangle\right\rangle \text { iff } l>l^{\prime} \\
& \left\langle l: e v \mid\left\langle l^{\prime}: e v^{\prime}, w\right\rangle\right\rangle=\left\langle l: e v, l^{\prime}: e v^{\prime}, w\right\rangle \quad \text { iff } l \leqslant l^{\prime}
\end{aligned}
$$

Note how the dynamic representation as vectors of labeled evidence nicely corresponds to the static effect row-types, in particular with regard to duplicate labels, which correspond to nested handlers at runtime. Here we see why we cannot swap the position of equal effect labels as we need the evidence to correspond to their actual order in the evaluation context. Inserting all evidence in a vector $w_{1}$ into another vector $w_{2}$ is defined inductively as following. We reuse the same notation of inserting a single evidence.

$$
\begin{aligned}
& \left\langle\left\langle\langle\rangle \mid w_{2}\right\rangle=w_{2}\right. \\
& \left\langle\left\langle l: e v, w_{1}\right\rangle \mid w_{2}\right\rangle=\left\langle l: e v \mid\left\langle w_{1} \mid w_{2}\right\rangle\right\rangle
\end{aligned}
$$

and evidence selection can be defined as:

$$
\begin{aligned}
& . l: \forall \mu . \operatorname{evv}\langle l \mid \mu\rangle \rightarrow \mathrm{ev} l \\
& \langle l: e v,\rangle . l=\mathrm{ev} \\
& \left\langle l^{\prime}: e v, w\right\rangle . l=\text { w. } \quad \text { iff } l \neq l^{\prime} \\
& \left\langle l^{\prime}=(\text { cannot happen) }\right.
\end{aligned}
$$




\subsection{Evidence Translation}

The evidence translation is already defined in Figure 5, in the gray parts of the rules. The full rules for expressions are of the form $\Gamma ; w \vdash e: \sigma \mid \epsilon \rightsquigarrow e^{\prime}$ where given a context $\Gamma$, the expression $e$ has type $\sigma$ with effect $\epsilon$. The rules take the current evidence vector $w$ for the effect $\epsilon$, of type evv $\epsilon$, and translate to an expression $e^{\prime}$ of System $\mathrm{F}^{e v}$.

The translation in itself is straightforward where we only need to ensure extra evidence is passed during applications and abstracted again on lambdas. The ABs rule abstracts fully over all evidence in a function as $\lambda^{\epsilon} z$ : evv $\epsilon, x: \sigma_{1} . e^{\prime}$, where the evidence vector is abstracted as $z$ and passed to its premise. Note that since we are translating, $z$ is not part of $\Gamma$ here (which scopes over $\mathrm{F}^{\epsilon}$ terms). The type rules for $\mathrm{F}^{e v}$, discussed below, do track such variables in the context. The dual of this is rule APP which passes the effect evidence $w$ as an extra argument to every application as $e_{1}^{\prime} w e_{2}^{\prime}$.

To prove preservation and coherence of the translation, we also include a translation rule for handle, even though we assume these are internal. Otherwise there are no surprises here and the main difficulty lies in the operational rules, which we discuss in detail in Section 4.4.

To prove additional properties about the translated programs, we define a more restricted set of typing rules directly over System $\mathrm{F}^{e v}$ in Figure 9 of the form $\Gamma ; w \Vdash e: \sigma \mid \epsilon$ (ignoring the gray parts), such that $\Gamma \vdash w: \operatorname{evv} \epsilon$. Using this, we prove that the translation is sound:

Theorem 4. (Evidence translation is Sound in $\mathrm{F}^{e v}$ )

If $\varnothing ;\langle\rangle \vdash e: \sigma \mid\langle\rangle \rightsquigarrow e^{\prime}$ then $\varnothing ;\langle\rangle \Vdash e^{\prime}:\lceil\sigma\rceil \mid\langle\rangle$.

\subsection{Correspondence}

The evidence translation maintains a strong correspondence between the effect types, the evidence vectors, and the evaluation contexts. To make this precise, we define the (reverse) extraction of all handlers in a context $\mathrm{E}$ as $\lceil\mathrm{E}\rceil$ where:

$$
\begin{aligned}
& \left\lceil\mathrm{F}_{1} \cdot \text { handle }\left.\right|_{m_{1}} ^{w_{1}} h_{1} \cdot \ldots \cdot \mathrm{F}_{n} \cdot \text { handle }\left.\right|_{m_{n}} ^{w_{n}} h_{n} \cdot \mathrm{F}\right\rceil=\left\langle l_{n}:\left(m_{n}, h_{n}\right)|\ldots| l_{1}:\left(m_{1}, h_{1}\right)\right\rangle \text { iff } h_{i}: \Sigma\left(l_{i}\right) \\
& \left\lceil\mathrm{F}_{1} \cdot \text { handle } m_{m_{1}}^{w_{1}} h_{1} \cdot \ldots \cdot \mathrm{F}_{n} \cdot \text { handle }_{m_{n}}^{w_{n}} h_{n} \cdot \mathrm{F}\right\rceil^{l}=\left\langle l_{n}, \ldots, l_{1}\right\rangle \\
& \left\lceil\mathrm{F}_{1} \cdot \text { handle }_{m_{1}}^{w_{1}} h_{1} \cdot \ldots \cdot \mathrm{F}_{n} \cdot \text { handle }_{m_{n}}^{w_{n}} h_{n} \cdot \mathrm{F}^{m}=\left\{m_{n}, \ldots, m_{1}\right\}\right.
\end{aligned}
$$

With this we can characterize the correspondence between the evaluation context and the evidence used at perform:

Lemma 6. (Evidence corresponds to the evaluation context)

If $\varnothing ; w \Vdash \mathrm{E}[e]: \sigma \mid \epsilon$ then for some $\sigma_{1}$ we have $\varnothing ;\langle\lceil\mathrm{E}\rceil \mid w\rangle \Vdash e: \sigma_{1} \mid\left\langle\lceil\mathrm{E}\rceil^{l} \mid \epsilon\right\rangle$, and $\varnothing ; w \Vdash \mathrm{E}: \sigma_{1} \rightarrow \sigma \mid \epsilon$.

Lemma 7. (Well typed operations are handled)

If $\varnothing ;\langle\rangle \Vdash \mathrm{E}[$ perform op $\bar{\sigma} v]: \sigma \mid\langle\rangle$ then $\mathrm{E}$ has the form $\mathrm{E}_{1} \cdot$ handle $\mathrm{e}_{m}^{w} h \cdot \mathrm{E}_{2}$ with op $\notin \operatorname{bop}\left(\mathrm{E}_{2}\right)$ and $o p \rightarrow f \in h$.

These brings us to our main theorem which states that the evidence passed to an operation corresponds exactly to the innermost handler for that operation in the dynamic evaluation context:

Theorem 5. (Evidence Correspondence)

If $\varnothing ;\langle\Downarrow \Vdash \mathrm{E}[$ perform op $\bar{\sigma} w v]: \sigma|\langle\rangle$ then $\mathrm{E}$ has the form $\mathrm{E}_{1} \cdot$ handle ${ }_{m}^{w^{\prime}} h \cdot \mathrm{E}_{2}$ with op $\in \Sigma(l)$, $o p \notin \operatorname{bop}\left(\mathrm{E}_{2}\right), o p \rightarrow f \in h$, and the evidence corresponds exactly to dynamic execution context such that $w . l=(m, h)$. 


\subsection{Operational Rules of System $F^{e v}$}

The operational rules for System $F^{e v}$ are defined in Figure 6. Since every application now always takes an evidence vector argument $w$ the new (app) and (handler) rules now only reduce when both arguments are present (and the syntax does not allow for partial evidence applications).

The (handler) rule differs from System $\mathrm{F}^{\epsilon}$ in two significant ways. First, it saves the current evidence in scope (passed as $w$ ) in the handle frame itself as handle ${ }_{m}^{w}$. Secondly, the evidence vector it passes on to its action is now extended with its own unique evidence, as $\langle l:(m, h) \mid w\rangle$.

In the (perform) rule, the operation clause $(o p \rightarrow f) \in h$ is now translated itself, and we need to pass evidence to $f$. Since it takes two arguments, the operation payload $x$ and its resumption $k$, the application becomes $(f[\bar{\sigma}] w x) w k$. The evidence we pass to $f$ is the evidence of the original handler context saved as handle ${ }^{w}$ in the (handler) rule. In particular, we should not pass the evidence $w^{\prime}$ of the operation, as that is the evidence vector of the context in which the operation itself evaluates (and an extension of $w$ ). In contrast, we evaluate each clause under their original context and need the evidence vector corresponding to that. In fact, we can even ignore the evidence vector $w^{\prime}$ completely for now as we only need to use it later for implementing optimizations.

\subsection{Guarded Context Instantiation and Scoped Resumptions}

The definition of the resumption $k$ in the (perform) rule differs quite a bit from the original definition in System $\mathrm{F}^{\epsilon}$ (Figure 2), which was:

$$
k=\lambda^{\epsilon} x: \sigma_{2}[\bar{\alpha}:=\bar{\sigma}] \text {. handle }{ }^{\epsilon} h \cdot \mathrm{E} \cdot x
$$

while the $\mathrm{F}^{e v}$ definition now uses:

$$
k=\operatorname{guard}^{w}\left(\text { handle }_{m}^{w} h \cdot \mathrm{E}\right)\left(\sigma_{2}[\bar{\alpha}:=\bar{\sigma}]\right)
$$

where we use a the new $\mathrm{F}^{e v}$ value term guard ${ }^{w} \mathrm{E} \sigma$. Since $k$ has a regular function type, it now needs to take an extra evidence vector, and we may have expected a more straightforward extension without needing a new guard rule, something like:

$$
k=\lambda^{\epsilon} z: \operatorname{evv} \epsilon, x: \sigma_{2}[\bar{\alpha}:=\bar{\sigma}] \text {. handle }{ }^{\epsilon} h \cdot \mathrm{E} \cdot x
$$

but then the question becomes what to do with that passed in evidence $z$ ? This is the point where it becomes more clear that resumptions are special and not quite like a regular lambda since they restore a captured context. In particular, the context $\mathrm{E}$ that is restored has already captured the evidence of the original context in which it was captured (as $w$ ), and thus may not match the evidence of the context in which it is resumed (as $z$ )!

The new guarded application rule makes this explicit and only restores contexts that are resumed under the exact same evidence, in other words, only scoped resumptions are allowed:

$$
\text { (guard } \left.{ }^{w} \mathrm{E} \sigma\right) w v \rightarrow \mathrm{E}[v]
$$

If the evidence does not match, the evaluation is stuck in $\mathrm{F}^{e v}$.

As an example of how this can happen, we return to our evil example in Section 2.2 which uses non-scoped resumptions to change the meaning of $o p_{1}$. Since we are now in a typed setting, we modify the example to return a data type of results to make everything well-typed:

$$
\begin{aligned}
& \text { data res }=\text { again }:(() \rightarrow\langle\text { one }\rangle \text { res }) \rightarrow \text { res } \\
& \quad \text { | done }: \text { int } \rightarrow \text { res } \\
& \Sigma=\left\{\text { one }:\left\{o p_{1}:() \rightarrow \text { int }\right\}, \text { evil }:\left\{o p_{\text {evil }}:() \rightarrow()\right\}\right\}
\end{aligned}
$$

with the following helper definitions:

$$
\begin{array}{lll}
h_{1}=\left\{o p_{1} \rightarrow \lambda x k . k 1\right. & & f(\text { again } k)=\text { handler } h_{2}\left(\lambda_{-} \cdot k()\right) ; 0 \\
h_{2}=\left\{o p_{1} \rightarrow \lambda x k . k 2\right\} & f(\text { done } x)=x \\
h_{\text {evil }}=\left\{o p_{\text {evil }} \rightarrow \lambda x k .(\text { again } k)\right\} &
\end{array}
$$


body $=$ perform $o p_{1}()$; perform $o p_{\text {evil }}()$; perform $o p_{1}()$; done 0

and where the main expression is evidence translated as:

$f$ (handler $h_{1}\left(\lambda_{-}\right.$. handler $h_{\text {evil }}\left(\lambda_{-}\right.$. body $\left.\left.)\right)\right)$

$\rightsquigarrow f\langle\rangle\rangle\left(\right.$ handler $h_{1}\langle\rangle\left(\lambda z\right.$, $_{-}$. handler $h_{\text {evil }} z$

$\left(\lambda z\right.$ : evv $\langle$ one, evil $\rangle, \ldots$ perform $o p_{1} z()$; perform $o p_{\text {evil }} z()$; perform $o p_{1} z()$; done 0$\left.\left.)\right)\right)$

Starting evaluation in the translated expression, we can now derive:

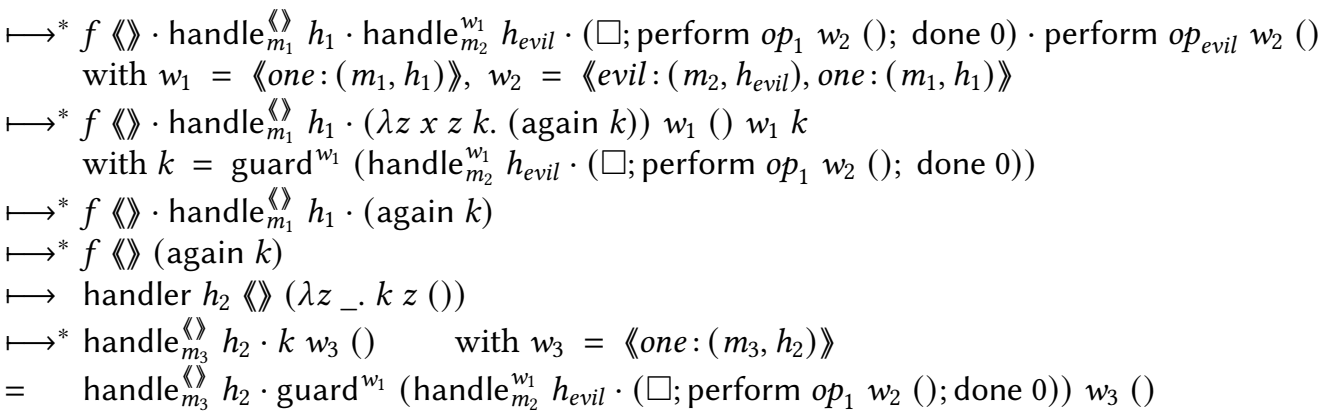

At this point, the guard rule gets stuck as we have captured the context originally under evidence $w_{1}$, but we try to resume with evidence $w_{3}$, and $w_{1}=\left\langle\right.$ one $\left.:\left(m_{1}, h_{1}\right)\right\rangle \neq\left\langle\right.$ one $\left.:\left(m_{3}, h_{2}\right)\right\rangle=w_{3}$.

If we allow the guarded context instantiation anyways we get into trouble when we try to perform $o p_{1}$ again:

$\longmapsto$ handle $m_{3} h_{2} \cdot$ handle $_{m_{2}}^{w_{1}} h_{\text {evil }} \cdot\left(()\right.$; perform $o p_{1} w_{2}()$; done 0$)$

$\longmapsto^{*}$ handle $m_{3}\langle\rangle h_{2} \cdot$ handle $_{m_{2}}^{w_{1}} h_{\text {evil }} \cdot(\square$; done 0$) \cdot$ perform $o p_{1} w_{2}()$

in that case the innermost handler for $o p_{1}$ is now $h_{2}$ while the evidence $w_{2} . l$ is $\left(m_{1}, h_{1}\right)$ and it no longer corresponds to the dynamic context! (and that would void our main correspondence Theorem 5 and in turn invalidate optimizations based on this).

\subsection{Uniqueness of Handlers}

It turns out that to guarantee coherence of the translation to plain polymorphic lambda calculus, as discussed in Section 5, we need to ensure that all $\mathrm{m}$ 's in an evaluation context are always unique. This is a tricky property; for example, uniqueness of markers does not hold for arbitrary $\mathrm{F}^{e v}$ expressions: markers may be duplicated inside lambdas outside of the evaluation context, and we can also construct an expression manually with duplicated markers, e.g. handle ${ }_{m}^{w} \cdot$ handle $_{m}^{w} \cdot e$. However, we can prove that if we only consider initial $\mathrm{F}^{e v}$ expressions without handle ${ }_{m}^{w}$, or any expressions reduced from that during evaluation, then it is guaranteed that all $m$ 's are always unique in the evaluation context - even though the (handler) rule introduces handle ${ }_{m}^{w}$ during evaluation, and the $(a p p)$ rule may duplicate markers.

Definition 1. (Handle-safe expressions)

A handle-safe $\mathrm{F}^{e v}$ expression is a well-typed closed expression that either (1) contains no handle ${ }_{m}^{w}$ term; or (2) is itself reduced from a handle-safe expression.

Theorem 6. (Uniqueness of handlers)

For any handle-safe $\mathrm{F}^{e v}$ expression $e$, if $e=\mathrm{E}_{1} \cdot$ handle $_{m_{1}}^{w_{1}} h \cdot \mathrm{E}_{2} \cdot$ handle $_{m_{2}}^{w_{2}} h \cdot e_{0}$, then $m_{1} \neq m_{2}$.

\subsection{Preservation and Coherence}

As exemplified above, the guard rule is also essential to prove the preservation of evidence typings under evaluation. In particular, we can show: 
Theorem 7. (Preservation of evidence typing)

If $\varnothing ;\langle\rangle \Vdash e_{1}: \sigma \mid\langle\rangle$ and $e_{1} \longmapsto e_{2}$, then $\varnothing ;\langle\rangle \Vdash e_{2}: \sigma \mid\langle\rangle$.

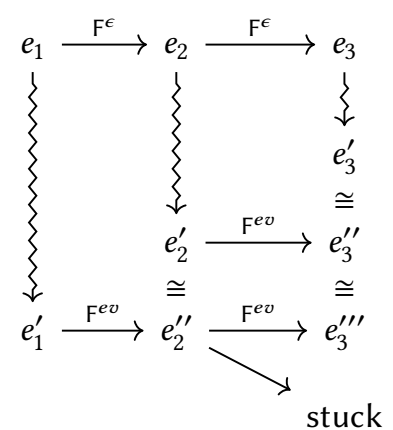

Fig. 7. Coherence

Even more important though is to show that our translation is coherent, that is, if we take an evaluation step in System $\mathrm{F}^{\epsilon}$, the evidence translated expression will take a similar step such that the resulting expression is again a translation of the reduced $\mathrm{F}^{\epsilon}$ expression:

\section{Theorem 8. (Evidence translation is coherent)}

If $\varnothing ;\langle\rangle \vdash e_{1}: \sigma \mid\langle\rangle \rightsquigarrow e_{1}^{\prime}$ and $e_{1} \longmapsto e_{2}$, and (due to preservation) $\varnothing ;\langle\rangle \vdash e_{2}: \sigma \mid\langle\rangle \rightsquigarrow e_{2}^{\prime}$, then there exists a $e_{2}^{\prime \prime}$, such that $e_{1}^{\prime} \longmapsto e_{2}^{\prime \prime}$ and $e_{2}^{\prime \prime} \cong e_{2}^{\prime}$.

Interestingly, the theorem states that the translated $e_{2}^{\prime}$ is only coherent under an equivalence relation $\cong$ to the reduced expression $e_{2}^{\prime \prime}$, as illustrated in Figure 7. The reason that $e_{2}^{\prime}$ and $e_{2}^{\prime \prime}$ are not directly equal is due to guard expressions only being generated by reduction. In particular, if we have a $\mathrm{F}^{\epsilon}$ reduction of the form:

handle ${ }^{\epsilon} h \cdot \mathrm{E} \cdot$ perform op $\bar{\sigma} v \longrightarrow f \bar{\sigma} v k \quad$ with $k=\lambda^{\epsilon} x: \sigma^{\prime}$. handle ${ }^{\epsilon} h \cdot \mathrm{E} \cdot x$ then the translation takes the following $\mathrm{F}^{e v}$ reduction:

handle $m_{m}^{w} h \cdot \mathrm{E}^{\prime} \cdot$ perform op $\lceil\bar{\sigma}\rceil w^{\prime} v^{\prime} \longrightarrow f^{\prime}\lceil\bar{\sigma}\rceil w v^{\prime} w k^{\prime}$ with $k^{\prime}=\operatorname{guard}^{w}\left(\right.$ handle $\left._{m}^{w} h^{\prime} \cdot \mathrm{E}^{\prime}\right) \sigma^{\prime}$ At this point the translation of $f \bar{\sigma} v k$ will be of the form $f^{\prime}\lceil\bar{\sigma}\rceil w^{\prime} v^{\prime} w^{\prime} k^{\prime \prime}$ where

$$
k^{\prime \prime}=\lambda^{\epsilon} z: \operatorname{evv} \epsilon, x \text {. handle }{ }^{\epsilon} h^{\prime} \cdot \mathrm{E}^{\prime \prime} \cdot x
$$

i.e. the resumption $k$ is translated as a regular lambda now and not as guard! Also, since $E$ is translated now under a lambda, the resulting $E^{\prime \prime}$ differs in all evidence terms $w$ in $E^{\prime}$ which will be $z$ instead.

However, we know that if the resumption $k^{\prime}$ is ever applied, the argument is either exactly $w$, in which case $\mathrm{E}^{\prime \prime}[z:=w]=\mathrm{E}^{\prime}$, or not equal to $w$ in which case the evidence translated program gets stuck. This is captured by $\cong$ relation which is the smallest transitive and reflexive congruence among well-typed $\mathrm{F}^{e v}$ expressions, up to renaming of unique markers, satisfying the EQ-GUARD rule, which captures the notion of guarded context instantiation.

$$
\left.\frac{e[z:=w] \cong \mathrm{E}[x]}{\lambda^{\epsilon} z, x: \sigma . e \cong \text { guard }^{w} \mathrm{E} \sigma} \text { [EQ-GUARD }\right]
$$

Now, is this definition of equivalence strong enough? Yes, because we can show that if two translated expressions are equivalent, then they stay equivalent under reduction (or get stuck):

Lemma 8. (Operational semantics preserves equivalence, or gets stuck) If $e_{1} \cong e_{2}$, and $e_{1} \longrightarrow e_{1}^{\prime}$, then either $e_{2}$ is stuck, or we have $e_{2}^{\prime}$ such that $e_{2} \longrightarrow e_{2}^{\prime}$ and $e_{1}^{\prime} \cong e_{2}^{\prime}$.

This establishes the full coherence of our evidence translation: if a translated expression reduces under $\mathrm{F}^{e v}$ without getting stuck, the final value is equivalent to the value reduced under System $\mathrm{F}^{\epsilon}$. Moreover, the only way an evidence translated expression can get stuck is if it uses non-scoped resumptions.

Note that the evidence translation never produces guard terms, so the translated expression can always take an evaluation step; however, subsequent evaluation steps may lead to guard terms, so after the first step, it may get stuck if a resumption is applied under a different handler context than where it was captured. 


$$
\begin{aligned}
& \text { Expressions } e::=v \mid \text { e e } \mid e[\sigma] \\
& \text { Values } \quad v::=x|\lambda x: \sigma . e| \Lambda \alpha^{k} . v \\
& \text { Context } \mathrm{F}::=\square|\mathrm{F} e| v \mathrm{~F} \mid \mathrm{F}[\sigma] \\
& \mathrm{E}::=\mathrm{F} \\
& (a p p) \quad\left(\lambda^{\epsilon} x: \sigma . e\right) v \longrightarrow e[x:=v] \\
& (\text { tapp }) \quad\left(\Lambda \alpha^{k} . v\right)[\sigma] \longrightarrow v[\alpha:=\sigma] \\
& \frac{x: \sigma \in \Gamma}{\Gamma \vdash_{\mathrm{F}} x^{\sigma}: \sigma}[\mathrm{FVAR}] \frac{\Gamma \vdash_{\mathrm{F}} v: \sigma}{\Gamma \vdash_{\mathrm{F}} \Lambda \alpha^{k} . v: \forall \alpha^{k} . \sigma}[\mathrm{FTABS}] \quad \frac{\Gamma, x: \sigma_{1} \vdash_{\mathrm{F}} e: \sigma_{2}}{\Gamma \vdash_{\mathrm{F}} \lambda x: \sigma_{1} . e: \sigma_{1} \rightarrow \sigma_{2}} \text { [FABS] } \\
& \frac{\Gamma \vdash_{\mathrm{F}} e_{1}: \sigma_{1} \rightarrow \sigma \quad \Gamma \vdash e_{2}: \sigma}{\Gamma \vdash_{\mathrm{F}} e_{1} e_{2}: \sigma}[\mathrm{FAPP}] \quad \frac{\Gamma \vdash_{\mathrm{F}} e: \forall \alpha^{k} . \sigma_{1} \vdash_{\mathrm{Wf}} \sigma: k}{\Gamma \vdash_{\mathrm{F}} e[\sigma]: \sigma_{1}[\alpha:=\sigma]} \text { [FTAPP] }
\end{aligned}
$$

Fig. 8. System $\mathrm{F}^{v}$ : explicitly typed (higher kinded) polymorphic lambda calculus with strict evaluation. Types as in Figure 3 with no effects on the arrows.

\section{TRANSLATION TO CALL-BY-VALUE POLYMORPHIC LAMBDA CALCULUS}

Now that we have a strong correspondence between evidence and the dynamic handler context, we can translate System $F^{e v}$ expressions all the way to the call-by-value polymorphic lambda calculus, System $\mathrm{F}^{v}$. This is important in practice as it removes all the special evaluation and type rules of algebraic effect handlers; this in turn means we can apply all the optimizations that regular compilers perform, like inlining, known case expansion, common sub-expression elimination etc. as usual without needing to keep track of effects. Moreover, it means we can compile directly to most common host platforms, like $\mathrm{C}$ or WebAssembly without needing a special runtime system to support capturing the evaluation context.

There has been previous work that performs such translation [Forster et al. 2019; Hillerström et al. 2017; Leijen 2017c], as well as various libraries that embed effect handlers as monads [Kammar et al. 2013; Wu et al. 2014] but without evidence translation such embeddings require either a sophisticated runtime system [Dolan et al. 2017 2015; Leijen 2017a], or are not quite as efficient as one might hope. The translation presented here allows for better optimization as it maintains evidence and has no special runtime requirements (it is just F!).

\subsection{Translating to Multi-Prompt Delimited Continuations}

As a first step, we show that we do not need explicit handle frames anymore that carry around the handler operations $h$, but can translate to multi-prompt delimited continuations [Brachthäuser and Schuster 2017]. Gunter, Rémy, and Riecke [1995] present the set and cupto operators for named prompts $m$ with the following "control-upto" rule:

set $m$ in $\cdot \mathrm{E}$. cupto $m$ as $k$ in $e \longrightarrow(\lambda k . e)(\lambda x . \mathrm{E} \cdot x) \quad m \notin\lceil\mathrm{E}\rceil^{m}$

This effectively exposes "shallow" multi-prompts: the continuation bound to $k$ is not delimited by $m$. For our purposes, we always need "deep" handling where the resumption evaluates under the same prompt again and we define

$$
\begin{aligned}
& \text { prompt }_{m} e \doteq \text { set } m \text { in } e \\
& \text { yield }_{m} f \doteq \text { cupto } m \text { as } k \text { in }(f(\lambda x . \text { set } m \text { in }(k x)))
\end{aligned}
$$

which gives us the following derived evaluation rule:

$$
\text { prompt }_{m} \cdot \mathrm{E} \cdot \text { yield }_{m} f \longrightarrow f\left(\lambda x \cdot \text { prompt }_{m} \cdot \mathrm{E} \cdot x\right) \quad m \notin\lceil\mathrm{E}\rceil^{m}
$$




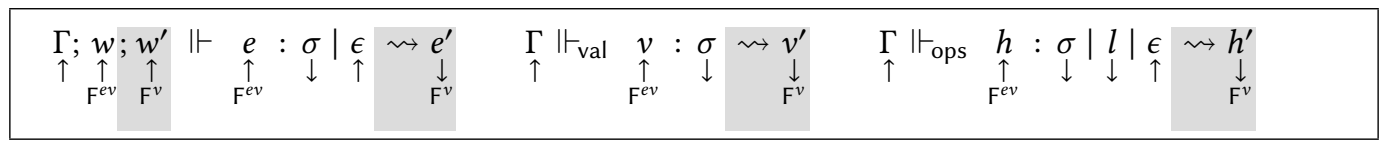

$$
\begin{aligned}
& \frac{x: \sigma \in \Gamma}{\Gamma \Vdash_{\mathrm{val}} x: \sigma \rightsquigarrow x}[\mathrm{MVAR}] \frac{\left(\Gamma, z: \operatorname{evv} \epsilon, x: \sigma_{1}\right) ; z ; z \Vdash e: \sigma_{2} \mid \epsilon \rightsquigarrow e^{\prime}}{\Gamma \Vdash_{\mathrm{val}} \lambda^{\epsilon} z: \operatorname{evv} \epsilon, x: \sigma_{1} \cdot e: \sigma_{1} \Rightarrow \epsilon \sigma_{2} \rightsquigarrow \lambda z x . e^{\prime}} \text { [MABs] } \\
& \frac{\Gamma \Vdash_{\text {val }} v: \sigma \rightsquigarrow v^{\prime}}{\Gamma \vdash_{\text {val }} \Lambda \alpha . v: \forall \alpha . \sigma \rightsquigarrow \Lambda \alpha . v^{\prime}}\left[\text { MTABs] } \frac{\Gamma \Vdash_{\text {val }} v: \sigma \rightsquigarrow v^{\prime}}{\Gamma ; w ; w^{\prime} \Vdash v: \sigma \mid \epsilon \rightsquigarrow p u r e[\lfloor\sigma]] v^{\prime}}[\text { MvAL] }\right. \\
& \frac{\Gamma ; w ; w^{\prime} \Vdash e: \forall \alpha . \sigma_{1} \mid \epsilon \rightsquigarrow e^{\prime}}{\Gamma ; w ; w^{\prime} \Vdash e[\sigma]: \sigma_{1}[\alpha:=\sigma] \mid \epsilon \rightsquigarrow e^{\prime} \triangleright(\lambda x \text {. pure }(x[\lfloor\sigma]]))} \text { [MтAрт] } \\
& \frac{\Gamma ; w ; w^{\prime} \Vdash e_{1}: \sigma_{2} \Rightarrow \epsilon \sigma\left|\epsilon \rightsquigarrow e_{1}^{\prime} \quad \Gamma ; w ; w^{\prime} \Vdash e_{2}: \sigma_{2}\right| \epsilon \rightsquigarrow e_{2}^{\prime}}{\Gamma ; w ; w^{\prime} \Vdash e_{1} w e_{2}: \sigma \mid \epsilon \rightsquigarrow e_{1}^{\prime} \triangleright\left(\lambda f .\left(e_{2}^{\prime} \triangleright f w^{\prime}\right)\right)} \text { [MAPP] } \\
& \frac{\Gamma ; w ; w^{\prime} \Vdash_{\text {ec }} \mathrm{E}: \sigma_{1} \rightarrow \sigma_{2} \mid \epsilon \rightsquigarrow e^{\prime} \quad \Gamma \Vdash_{\text {val }} w: \operatorname{evv} \epsilon \rightsquigarrow w^{\prime}}{\Gamma \Vdash_{\text {val }} \text { guard }^{w} \mathrm{E} \sigma_{1}: \sigma_{1} \Rightarrow \epsilon \sigma_{2} \rightsquigarrow \text { guard } w^{\prime} e^{\prime}} \text { [MGUARD] } \\
& o p: \forall \bar{\alpha} . \sigma_{1} \rightarrow \sigma_{2} \in \Sigma(l)
\end{aligned}
$$

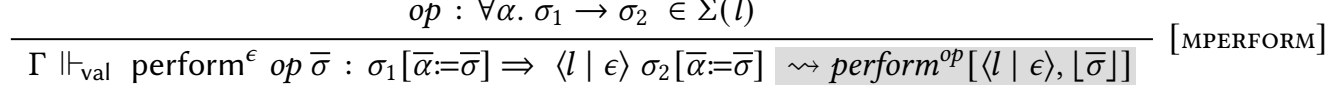

$$
\begin{aligned}
& \begin{array}{c}
o p_{i}: \forall \bar{\alpha} . \sigma_{1} \rightarrow \sigma_{2} \in \sum(l) \quad \bar{\alpha} \pitchfork \mathrm{ftv}(\epsilon \sigma) \\
\Gamma \Vdash_{\mathrm{val}} f_{i}: \forall \bar{\alpha} . \sigma_{1} \Rightarrow \epsilon\left(\sigma_{2} \Rightarrow \epsilon \sigma\right) \Rightarrow \epsilon \sigma \rightsquigarrow f_{i}^{\prime} \\
\frac{\Gamma \vdash_{\mathrm{ops}}\left\{o p_{1} \rightarrow f_{1}, \ldots, o p_{n} \rightarrow f_{n}\right\}: \sigma|l| \epsilon \rightsquigarrow\left\{o p_{1} \rightarrow f_{1}^{\prime}, \ldots, o p_{n} \rightarrow f_{n}^{\prime}\right\}}{\text { [Mops] }}
\end{array} \\
& \frac{\Gamma \Vdash_{\text {ops }} h: \sigma|l| \epsilon \rightsquigarrow h^{\prime}}{\Gamma \Vdash_{\text {val }} \text { handler }^{\epsilon} h:(() \Rightarrow\langle l \mid \epsilon\rangle \sigma) \Rightarrow \epsilon \sigma \rightsquigarrow \operatorname{handler}^{l}[\epsilon,\lfloor\sigma\rfloor] h^{\prime}} \text { [MHANDLER] } \\
& \Gamma \Vdash_{\text {ops }} h: \sigma|l| \epsilon \rightsquigarrow h^{\prime} \\
& \frac{\Gamma ;\langle l:(m, h) \mid w\rangle ;\left\langle\left\langle l:\left(m, h^{\prime}\right) \mid w^{\prime}\right\rangle \Vdash e: \sigma\right|\langle l \mid \epsilon\rangle \rightsquigarrow e^{\prime}}{\Gamma ; w ; w^{\prime} \Vdash \text { handle }_{m}^{w} h e: \sigma \mid \epsilon \rightsquigarrow \operatorname{prompt}[\epsilon,\lfloor\sigma\rfloor] m w^{\prime} e^{\prime}} \text { [MHANDLE] }
\end{aligned}
$$

Fig. 9. Monadic translation to System- $\mathrm{F}^{v}$. (( $\left.\triangleright\right)$ is monadic bind).

Additionally, our control operators also need to take evidence $w$ into account and use guard instead of a plain lambda to apply the resumption, i.e.,

$$
\operatorname{prompt}_{m}^{w} \cdot \mathrm{E} \cdot \text { yield }_{m} f \longrightarrow f w\left(\text { guard }^{w}\left(\operatorname{prompt}_{m}^{w} \cdot \mathrm{E}\right)\right) \quad m \notin\lceil\mathrm{E}\rceil^{m}
$$

Therefore, we take prompt and yield as primitive control operators with the above evaluation rule.

Using the correspondence property (Theorem 5), we can use evidence to locally inspect the handler on perform and no longer need to keep it in the handle frame. We can now translate both perform op $v w$ and handle ${ }_{m}^{w} h$ in terms of the simpler yield ${ }_{m}$ and $\operatorname{prompt}_{m}^{w}$, as:

$$
\begin{aligned}
& \left\lceil\text { handle }{ }_{m}^{w} h\right\rceil=\operatorname{prompt}_{m}^{w} \\
& \left\lceil\text { perform op } w^{\prime} v\right\rceil=\operatorname{yield}_{m}(\lambda w k . f w v w k) \quad \text { with }(m, h)=w^{\prime} . l \text { and }(o p \rightarrow f) \in h
\end{aligned}
$$


We prove that this is a sound interpretation of effect handling:

Theorem 9. (Evidence Translation to Multi-Prompt Delimited Continuations is Sound) For any evaluation step $e_{1} \longmapsto e_{2}$ in $\mathrm{F}^{e v}$, we have $\left\lceil e_{1}\right\rceil \longmapsto^{*}\left\lceil e_{2}\right\rceil$ with multi-prompt delimited continuations.

Dolan et al. [2015] describe the multi-core OCaml runtime system with split stacks; in such setting we could use the pointers to a split point as markers $m$, and directly yield to the correct handler with constant time capture of the context.

\subsection{Monadic Multi-Prompt Translation to System $\mathrm{F}^{v}$}

With the relation to multi-prompt delimited control established, we can now translate $\mathrm{F}^{e v}$ to $\mathrm{F}^{v}$ in a monadic style, where we use standard techniques [Dyvbig et al. 2007] to implement the delimited control as a monad. Assuming notation for data types and matching, we can define a multi-prompt monad mon as follows:

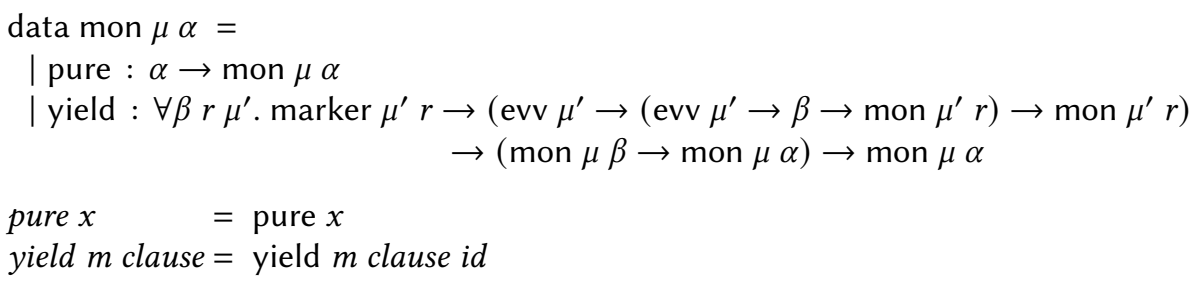

The pure case is used for value results, while the yield implements yielding to a prompt. A yield $m f$ cont has three arguments, (1) the marker $m$ : marker $\mu^{\prime} r$ bound to a prompt in some context with effect $\mu^{\prime}$ and answer type $r$; (2) the operation clause which receives the resumption (of type $\beta \rightarrow$ mon $\mu^{\prime} r$ ) where $\beta$ is the type of the operation result; and finally (3) the current continuation cont which is the runtime representation of the context. When binding a yield, the continuation keeps being extended until the full context is captured:
$(f \circ g) x$
$=f(g x)$
(function composition)
$(f \cdot g) x$
$=g x \triangleright f$
(Kleisli composition)
(pure $x) \triangleright g \quad=g x$
(monadic bind)
(yield $m f$ cont $) \triangleright g=$ yield $m f(g \bullet$ cont $)$

The hoisting of yields corresponds closely to operation hoisting as described by Bauer and Pretnar [2015b]. The prompt operation has three cases to consider:

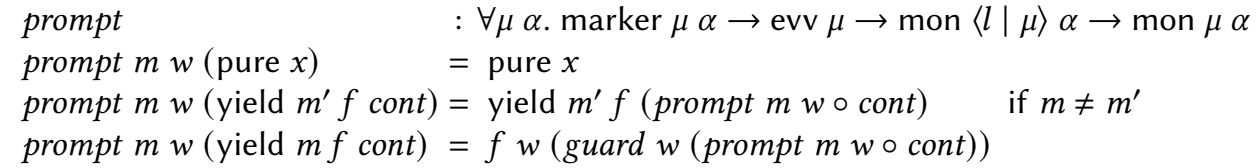

In the pure case, we are at the (value) rule and return the result as is. If we find a yield that yields to another prompt we also keep yielding but remember to restore our prompt when resuming in its current continuation, as (prompt $m w \circ$ cont). The final case is when we yield to the prompt itself, in that case we are in the (yield) transition and continue with $f$ passing the context evidence $w$ and a guarded resumption ${ }^{3}$.

The guard operation simply checks if the evidence matches and either continues or gets stuck: guard $w_{1}$ cont $w_{2} x=$ if $\left(w_{1}==w_{2}\right)$ then cont (pure $\left.x\right)$ else stuck

\footnotetext{
${ }^{3}$ Typing the third case needs a dependent match on the markers $m^{\prime}$ : marker $\mu^{\prime} r$ and $m=$ marker $\mu \alpha$ where their equality implies $\mu=\mu^{\prime}$ and $r=\alpha$. This can be done in Haskell with the Equal GADT, or encoded in $\mathrm{F}^{v}$ using explicit equality witnesses [Baars and Swierstra 2002].
} 
Note that due to the uniqueness property (Theorem 6 ) we can check the equality $w_{1}==w_{2}$ efficiently by only comparing the markers $m$ (and ignoring the handlers). The handle and perform can be translated directly into prompt and yield as shown in the previous section, where we generate a handler $^{l}$ definition per effect $l$, and a perform ${ }^{o p}$ for every operation:

$$
\begin{array}{ll}
\text { handler }^{l} & : \forall \mu \alpha . \text { hnd }^{l} \mu \alpha \rightarrow \operatorname{evv} \mu \rightarrow(\operatorname{evv}\langle l \mid \mu\rangle \rightarrow() \rightarrow \operatorname{mon}\langle l \mid \mu\rangle \alpha) \rightarrow \operatorname{mon} \mu \alpha \\
\text { perform }^{o p} \quad: \forall \mu \bar{\alpha} . \operatorname{evv}\langle l \mid \mu\rangle \rightarrow \sigma_{1} \rightarrow \operatorname{mon}\langle l \mid \mu\rangle \sigma_{2} \quad \text { with op }: \forall \bar{\alpha} . \sigma_{1} \rightarrow \sigma_{2} \in \Sigma(l) \\
\text { handler }^{l} h w f & =\text { freshm }(\lambda m \rightarrow \text { prompt } m w(f\langle l:(m, h) \mid w\rangle())) \\
\text { perform }^{o p} w x & =\text { let }(m, h)=\text { w.l in yield } m(\lambda w k .((h . o p) w x \triangleright(\lambda f . f w k)))
\end{array}
$$

The handler creates a fresh marker and passes on new evidence under a new prompt. The perform can now directly select the evidence $(m, h)$ from the passed evidence vector and yield to $m$ directly. The function passed to yield is a bit complex since each operation clause is translated normally and has a nested monadic type, so we need to bind the first partial application to $x$ before passing the continuation $k$.

Finally, for every effect signature $l: \operatorname{sig} \in \sum$ we declare a corresponding data type $\mathrm{hnd}^{l} \epsilon r$ that is a record of operation clauses:

$$
\begin{aligned}
l & :\left\{o p_{1}: \forall \bar{\alpha}_{1}, \sigma_{1} \rightarrow \sigma_{1}^{\prime}, \ldots, o p_{n}: \forall \bar{\alpha}_{n} . \sigma_{n} \rightarrow \sigma_{n}^{\prime}\right\} \\
& \rightsquigarrow \text { data hnd } \text { h }^{l} \mu r=\text { hnd }^{l}\left\{o p_{1}: \forall \bar{\alpha}_{1} \text {. op } \sigma_{1} \sigma_{1}^{\prime} \mu r, \ldots, o p_{n}: \forall \bar{\alpha}_{n} \text {. op } \sigma_{n} \sigma_{n}^{\prime} \mu r\right\}
\end{aligned}
$$

where operations op are a type alias defined as:

$$
\text { alias op } \alpha \beta \mu r \doteq \operatorname{evv} \mu \rightarrow \alpha \rightarrow \operatorname{mon} \mu(\operatorname{evv} \mu \rightarrow(\operatorname{evv} \mu \rightarrow \beta \rightarrow \operatorname{mon} \mu r) \rightarrow \operatorname{mon} \mu r)
$$

With these definitions in place, we can do a straightforward type directed translation from $\mathrm{F}^{e v}$ to $\mathrm{F}^{v}$ by just lifting all operations into the prompt monad, as shown in Figure 9. Types are translated by making all effectful functions monadic:

$$
\begin{array}{lll}
\lfloor\forall \bar{\alpha} . \sigma\rfloor=\forall \bar{\alpha} .\lfloor\sigma\rfloor & & \left\lfloor\sigma_{1} \Rightarrow \epsilon \sigma_{2}\right\rfloor=\operatorname{evv} \epsilon \rightarrow\left\lfloor\sigma_{1}\right\rfloor \rightarrow \operatorname{mon} \epsilon\left\lfloor\sigma_{2}\right\rfloor \\
\lfloor\alpha\rfloor=\alpha & \left\lfloor c \sigma_{1} \ldots \sigma_{n}\right\rfloor=c\left\lfloor\sigma_{1}\right\rfloor \ldots\left\lfloor\sigma_{n}\right\rfloor
\end{array}
$$

We prove that these definitions are correct, and that the resulting translation is fully coherent, where a monadic program evaluates to the same result as a direct evaluation in $\mathrm{F}^{e v}$.

Theorem 10. (Monadic Translation is Sound)

If $\varnothing ;\langle\rangle\rangle ;\langle\rangle \Vdash e: \sigma \mid\langle\rangle \rightsquigarrow e^{\prime}$, then $\varnothing \vdash_{\mathrm{F}} e^{\prime}: \operatorname{mon}\langle\rangle\lfloor\sigma\rfloor$.

Theorem 11. (Coherence of the Monadic Translation)

If $\varnothing ;\left\langle\langle\rangle ;\langle\rangle \Vdash e_{1}: \sigma\right|\langle\rangle \rightsquigarrow e_{1}^{\prime}$ and $e_{1} \longrightarrow e_{2}$, then $\varnothing ;\left\langle\langle\rangle ;\langle\rangle \Vdash e_{2}: \sigma\right|\langle\rangle \rightsquigarrow e_{2}^{\prime}$ where $e_{1}^{\prime} \longrightarrow{ }^{*} e_{2}^{\prime}$.

Together with earlier results we establish full soundness and coherence from the original typed effect handler calculus $\mathrm{F}^{\epsilon}$ to the evidence based monadic translation into plain call-by-value polymorphic lambda calculus $\mathrm{F}^{v}$. See Figure 10 for how our theorems relate these systems to each other.

\section{OPTIMIZATIONS}

With a fully coherent evidence translation to plain polymorphic lambda calculus in hand, we can now apply various transformations in that setting to optimize the resulting programs.

\subsection{Partially Applied Handlers}

In the current perform ${ }^{o p}$ implementation, we yield with a function that takes evidence $w$ to pass on to the operation clause $f$, as:

$$
\lambda w k .((h . o p) w x \triangleright(\lambda f . f w k))
$$




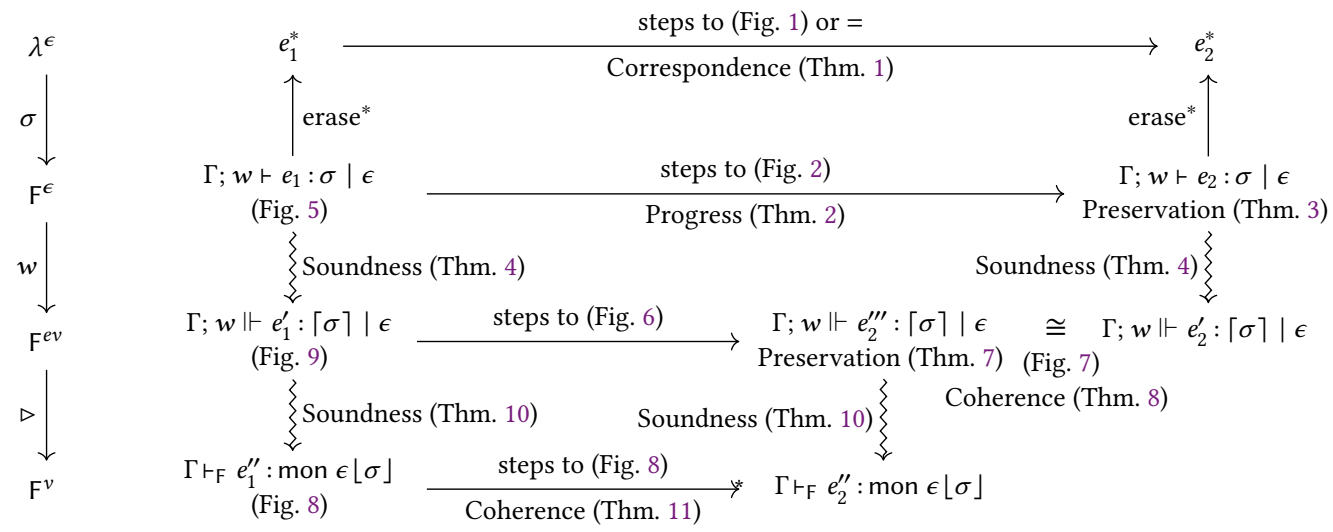

Fig. 10. An overview how the various theorems establish the relations between the different calculi

However, the $w$ that is going to be passed in is always that of the handle ${ }_{m}^{w}$ frame, as explained in Section 4.4. When we instantiate the handle ${ }_{m}^{w}$ we can in principle map the $w$ in advance over all operation clause so these can be partially evaluated over the evidence vector:

$$
\begin{aligned}
& \text { handler }^{l} h w f \quad=\text { freshm }\left(\lambda m \rightarrow \text { prompt } m w \left(f\left\langle l:\left(m, \operatorname{pmap}^{l} w h|w\rangle()\right)\right)\right.\right. \\
& \operatorname{pmap}^{l} w\left(\text { hnd }^{l} f_{1} \ldots f_{n}\right)=\operatorname{phnd}^{l}\left(\text { partial } w f_{1}\right) \ldots\left(\text { partial } w f_{n}\right) \\
& \text { partial } \quad: \operatorname{evv} \mu \rightarrow \text { op } \alpha \beta \mu r \rightarrow \operatorname{pop} \alpha \beta \mu r \\
& \text { partial } w f \quad=\lambda x k .\left(f w x \triangleright\left(\lambda f^{\prime} . f^{\prime} w k\right)\right)
\end{aligned}
$$

The pmap ${ }^{l}$ function creates a new handler data structure $p h n d^{l}$ where every operation is now partially applied to the evidence which results in simplified type for each operation (as expressed by the pop type alias):

$$
\text { alias pop } \alpha \beta \mu r \doteq \alpha \rightarrow(\operatorname{evv} \mu \rightarrow \beta \rightarrow \operatorname{mon} \mu r) \rightarrow \text { mon } \mu r
$$

The perform is now simplified as well as it no longer needs to bind the intermediate application:

$$
\text { perform }^{o p} w x=\text { let }(m, h)=w . l \text { in yield } m(\lambda k \text {. (h.op) } x k)
$$

Finally, the prompt case where the marker matches no longer needs to pass evidence as well:

$$
\text { prompt } m w(\text { yield } m f \text { cont })=f(\text { guard } w(\text { prompt } m w \circ \text { cont }))
$$

By itself, the impact of this optimization will be modest, just allowing inlining of evidence in $f$ clauses, but it opens up the way to do tail resumptive operations in-place.

\subsection{Evaluating Tail Resumptive Operations In Place}

In practice, almost all important effects are tail-resumptive. The main exceptions we know of are asynchronous I/O (but that is dominated by I/O anyways) and the ambiguity effect for resuming multiple times. As such, we expect the vast majority of operations to be tail-resumptive, and being able to optimize them well is important. We can extend the partially evaluated handler approach to optimize tail resumptions as well. First we extend the pop type to be a data type that signifies if an operation clause is tail resumptive or not:

$$
\begin{aligned}
\text { data pop } \alpha \beta \mu r & =\text { tail }:(\alpha \rightarrow \operatorname{mon} \mu \beta) \rightarrow \operatorname{pop} \alpha \beta \mu r \\
& \mid \text { normal }:(\alpha \rightarrow(\operatorname{evv} \mu \rightarrow \beta \rightarrow \operatorname{mon} \mu r) \rightarrow \operatorname{mon} \mu r) \rightarrow \operatorname{pop} \alpha \beta \mu r
\end{aligned}
$$


The partial function now creates tail terms for any clause $f$ that the compiler determined to be tail resumptive (i.e. of the form $\lambda x k . k e$ with $k \notin \mathrm{fv}(e)$ ):

$$
\begin{array}{ll}
\text { partial } w f=\operatorname{tail}\left(\lambda x .\left(f w x \triangleright\left(\lambda f^{\prime} \cdot f^{\prime} w \text { pure }\right)\right)\right. & \text { if } f \text { is tail resumptive } \\
\text { partial } w f=\operatorname{normal}\left(\lambda x k .\left(f w x \triangleright\left(\lambda f^{\prime} \cdot f^{\prime} w k\right)\right)\right. & \text { otherwise }
\end{array}
$$

Note that even if $f$ is tail resumptive, it may still use $x$. Moreover, since $f$ has already captured its evidence $w$, it can still perform operations itself.

Instead of passing in an "real" resumption function $k$, we just pass pure directly, leading to

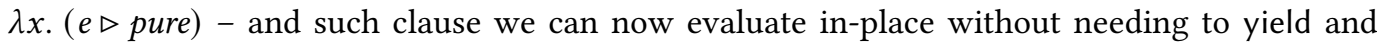
capture our resumption context explicitly. The perform ${ }^{o p}$ can directly inspect the form of the operation clause from its evidence, and evaluate in place when possible:

$$
\text { perform }^{o p} w x=\text { let }(m, h)=w . l \text { in case h.op of } \mid \begin{aligned}
& \text { tail } f \rightarrow f x \\
& \mid \text { normal } f \rightarrow \text { yield } m(f x)
\end{aligned}
$$

Moreover, if a known handler is applied over some expression, regular optimizations like inlining and known-case evaluation, can often inline the operations fully. As everything has been translated to regular functions and regular data types without any special evaluation rules, there is no need for special optimization rules for handlers either.

\subsection{Using Constant Offsets in Evidence Vectors}

The perform ${ }^{o p}$ operation is now almost as efficient as a virtual method call for tail resumptive operations (just check if it is tail and do in indirect call), except that it still needs to do a dynamic lookup for the evidence as $w . l$.

The idea is to take advantage of the canonical order of the evidence in a vector, where the location of the evidence in a vector of a closed effect type is fully determined. In particular, for any evidence vector $w$ of type evv $\langle l \mid \epsilon\rangle$ where $\epsilon$ is closed, we can replace $w . l$ by a direct index $w[o f s]$ where $(l$ in $\epsilon)=$ ofs, defined as:

$$
\begin{array}{lll}
l \text { in }\langle\rangle & =0 & \\
l \text { in }\left\langle l^{\prime} \mid \epsilon\right\rangle=l \text { in } \epsilon & & \text { iff } l \leqslant l^{\prime} \\
l \text { in }\left\langle l^{\prime} \mid \epsilon\right\rangle=1+(l \text { in } \epsilon) & \text { iff } l>l^{\prime}
\end{array}
$$

This means for any functions with a closed effect, the offset of all evidence is constant. Only functions that are polymorphic in the effect tail need to index dynamically. Details are beyond the scope of this paper and are left to future work, but we believe that even in those cases we can index by a direct offset: following the same approach as TREX [Gaster and Jones 1996], we can use qualified types internally to propagate $(l$ in $\mu)$ constraints where the "dictionary" is simply the offset in the evidence vector (and these constraints can be hidden from the user as we can always solve them).

\subsection{Reducing Continuation Allocation}

The monadic translation still produces inefficiencies as it captures the continuation at every point where an operation may yield. For example, when calling an effectful function foo, as in $x \leftarrow$ foo (); $e$, the monadic translation produces a bind which takes an allocated lambda as a second argument to represent the continuation $e$ explicitly, as foo ()$\triangleright(\lambda x . e)$.

First of all, we can do a selective monadic translation [Leijen 2017c] where we leave out the binds if the effect of a function can be guaranteed to never produce a yield, e.g. total functions (like arithmetic), all effects provided by the host platform (like I/O), and all effects that are statically guaranteed to be tail resumptive (called linear effects). It turns out that many (leaf) functions satisfy this property so this removes the vast majority of binding. 
Secondly, since we expect the vast majority of operations to be tail resumptive, almost always the effectful functions will not yield at all. It therefore pays off to always inline the bind operation and perform a direct match on the result and inline the continuation directly, e.g., we can expand $x \leftarrow$ foo (); $e$ to:

$$
\begin{gathered}
\text { case foo }() \text { of } \mid \text { yield } m f \text { cont } \rightarrow \text { yield } m f((\lambda x . e) \bullet \text { cont }) \\
\text { | pure } x \rightarrow e
\end{gathered}
$$

This can be done very efficiently, and is close to what a $\mathrm{C}$ or Go programmer would write: returning a (yielding) flag from every function and checking the flag before continuing. Of course, this is also a dangerous optimization as it duplicates the expression $e$, and more research is needed to evaluate the impact of code duplication and finding a good inlining heuristic.

As a closing remark, the above optimization is why we prefer the monadic approach over continuation passing style (CPS). With CPS, our example would pass the continuation directly as foo () $(\lambda x . e)$. This style may be more efficient if one often yields (as the continuation is more efficiently composed versus bubbling up through the binds [Ploeg and Kiselyov 2014]) but it prohibits our optimization where we can inspect the result of foo (without knowing its implementation) and to only allocate a continuation if it is really needed.

\subsection{Implementation}

We have an initial implementation of the evidence-passing translation in the Koka language [Leijen 2019] using the JavaScript backend ${ }^{4}$. The original runtime implementation uses a combination of CPS translation [Leijen 2017c] and an internal shadow stack of handlers where the operations propagate through this stack. The runtime part is about 1000 lines of JavaScript. The new implementation based on evidence-passing translation requires just a few primitives though (about 100 lines of JavaScript to handle evidence vectors efficiently).

While a systematic evaluation of efficient implementation strategies using evidence translation is beyond the scope of this paper, the initial benchmark results look promising. In particular, using benchmarks by Kiselyov et al. [2013] and Kammar et al. [2013], we compiled the same source with the original compiler (runtime) and with the new evidence translating compiler (evidence), and compare against the direct implementation of the benchmark that uses no handlers. We summarize the benchmark results in the following table. The table presents the relative speed of each implementation compared to the runtime compiler ${ }^{5}$.

\begin{tabular}{l|r|r|r|l} 
& runtime & evidence & direct & description \\
\hline counter & $1.00 \times$ & $1.94 \times$ & $2.14 \times$ & A counter in a loop. \\
count-mod5 & $1.00 \times$ & $1.28 \times$ & $0.83 \times$ & $\begin{array}{l}\text { Fold over a list and increment a counter on } \\
\text { every 5th element. }\end{array}$ \\
layered & $1.00 \times$ & $2.23 \times$ & $2.34 \times$ & $\begin{array}{l}\text { Use a state handler above five other reader } \\
\text { effect handlers. } \\
\text { nqueens }\end{array}$ \\
$1.00 \times$ & $46.09 \times$ & $76.20 \times$ & The n-queens problem.
\end{tabular}

In counter, evidence is almost twice as fast as runtime, executing the tail resumptive increment in place. It is also getting close to direct. In count-mod5, the improvement is more modest. Note that direct is slower here due to the need to propagate the counter explicitly as an extra argument. The layered benchmark can impact performance if searching linearly for a handler. This time evidence is more than twice as fast, and again close to direct. Finally, on nqueens, evidence is much faster

\footnotetext{
${ }^{4}$ In the dev-ev branch in the Koka repository [Leijen 2019].

${ }^{5}$ The relative speed is adjusted for any speed differences between the direct versions with the runtime and evidence compiler This compensates for any other differences in optimizations between the compiler versions (where the evidence compiler is usually a tad faster on the direct version).
} 
and about two thirds the speed of direct. Since evidence translation as such does not speed up the capturing and restoring of backtracking resumptions as used in nqueens, this result is a bit surprising. We conjecture that the explicit representation of continuations and evidence also helps the JavaScript compiler to optimize well, while the internal shadow stack handling in the runtime implementation may prohibit such optimizations here.

\section{RELATED WORK}

Throughout the paper, we compare with most related work, inline. Here, we discuss closely relevant work related to explicit passing of handlers.

Recent work by Biernacki et al. [2019] introduces labeled effect handlers, allowing handlers to being explicitly referred to by name; the generative semantics with labels $l$ is similar to our runtime markers $m$, but these labels are not guaranteed to be unique in the evaluation context (and they use the innermost handler in such case). Biernacki et al. also distinguish between the generative handler (as handle $e_{a}$ ), and the expression form handle (as handle $_{l}$ ).

Brachthäuser et al. use capability passing to perform operations directly on a specific handler [Brachthäuser and Schuster 2017; 2018; Brachthäuser et al. 2020; Schuster et al. 2020]. This is also similar to the work of Zhang and Myers [2019] where handlers are passed by name as well. While they pass evidence individually for each handler, we uniformly pass a vector of handlers. Both of these approaches can be viewed as programming within an explicit evidence passing calculus.

The work by Forster et al. [2019] is close to our work as it shows how delimited control, monads, and effect handlers can express each other. They show in particular a monadic semantics for effect handlers, but also prove that there does not exist a typed translation in their monomorphic setting. They conjecture a polymorphic translation may exist, and this paper proves that such translation is indeed possible.

Finally, we present a Haskell library [Xie and Leijen 2020] of effect handlers based on the evidence translation technique as described in this paper. While in this paper we encode effects using a row type system, the Haskell library encodes effects using a combination of a type list and type class constraints. It is shown that the library delivers good performance, and tends to outperform monads and alternative Haskell libraries when combining multiple effects.

\section{CONCLUSION}

We have shown a full formal and coherent translation from a polymorphic core calculus for effect handlers $\left(\mathrm{F}^{\epsilon}\right)$ to a polymorphic lambda calculus $\left(\mathrm{F}^{v}\right)$ based on evidence translation (through $\mathrm{F}^{e v}$ ), and we have characterized the relation to multi-prompt delimited continuations precisely. Besides giving a new framework to reason about semantics of effect handlers, we are also hopeful that these techniques will be used to create efficient implementations of effect handlers in practice. Moreover, from a language design perspective, we expect that the restriction to scoped resumptions will be more widely adopted. Currently we are working on an efficient backend for the Koka language to $\mathrm{C}$ code using evidence translation. As part of that work, we are investigating an extension of evidence translation that can potentially handle non-scoped resumptions as well. As future work, we are also interested in a systematic evaluation of efficient implementation strategies using evidence translation.

\section{ACKNOWLEDGMENTS}

We would like to thank Matthew Fluet, and other anonymous reviewers, for their detailed and insightful feedback on earlier versions of this paper. 


\section{REFERENCES}

Arthur I. Baars, and S. Doaitse Swierstra. 2002. Typing Dynamic Typing. In Proceedings of the Seventh ACM SIGPLAN International Conference on Functional Programming, 157-166. ICFP'02. Pittsburgh, PA, USA. doi:10.1145/581478.581494.

Andrej Bauer, and Matija Pretnar. 2014. An Effect System for Algebraic Effects and Handlers. Logical Methods in Computer Science 10 (4).

Andrej Bauer, and Matija Pretnar. 2015a. Programming with Algebraic Effects and Handlers. fournal of Logical and Algebraic Methods in Programming 84 (1). Elsevier: 108-123.

Andrej Bauer, and Matija Pretnar. 2015b. Programming with Algebraic Effects and Handlers. F. Log. Algebr. Meth. Program. 84 (1): 108-123. doi:10.1016/j.jlamp.2014.02.001.

Dariusz Biernacki, Maciej Piróg, Piotr Polesiuk, and Filip Sieczkowski. Dec. 2017. Handle with Care: Relational Interpretation of Algebraic Effects and Handlers. Proc. ACM Program. Lang. 2 (POPL'17 issue): 8:1-8:30. doi:10.1145/3158096.

Dariusz Biernacki, Maciej Piróg, Piotr Polesiuk, and Filip Sieczkowski. Dec. 2019. Binders by Day, Labels by Night: Effect Instances via Lexically Scoped Handlers. Proc. ACM Program. Lang. 4 (POPL). Association for Computing Machinery, New York, NY, USA. doi:10.1145/3371116.

Jonathan Immanuel Brachthäuser, and Philipp Schuster. Oct. 2017. Effekt: Extensible Algebraic Effects in Scala. In Scala'17. Vancouver, CA.

Jonathan Immanuel Brachthäuser, Philipp Schuster, and Klaus Ostermann. Oct. 2018. Effect Handlers for the Masses. Proc. ACM Program. Lang. 2 (OOPSLA). Association for Computing Machinery, New York, NY, USA. doi:10.1145/3276481.

Jonathan Immanuel Brachthäuser, Philipp Schuster, and Klaus Ostermann. 2020. Effekt: Lightweight Effect Polymorphism for Handlers. Technical Report. University of Tübingen, Germany.

Jonathan Immanuel Brachthäuser, Philipp Schuster, and Klaus Ostermann. 2020. Effekt: Capability-Passing Style for Typeand Effect-Safe, Extensible Effect Handlers in Scala. fournal of Functional Programming. Cambridge University Press.

Stephen Dolan, Spiros Eliopoulos, Daniel Hillerström, Anil Madhavapeddy, KC Sivaramakrishnan, and Leo White. May 2017. Concurrent System Programming with Effect Handlers. In Proceedings of the Symposium on Trends in Functional Programming. TFP'17.

Stephen Dolan, Leo White, KC Sivaramakrishnan, Jeremy Yallop, and Anil Madhavapeddy. Sep. 2015. Effective Concurrency through Algebraic Effects. In OCaml Workshop.

R Kent Dyvbig, Simon Peyton Jones, and Amr Sabry. 2007. A Monadic Framework for Delimited Continuations. Journal of Functional Programming 17 (6). Cambridge University Press: 687-730. doi:10.1017/S0956796807006259.

Yannick Forster, Ohad Kammar, Sam Lindley, and Matija Pretnar. 2019. On the Expressive Power of User-Defined Effects: Effect Handlers, Monadic Reflection, Delimited Control. fournal of Functional Programming 29. Cambridge University Press: 15. doi:10.1017/S0956796819000121.

Ben R. Gaster, and Mark P. Jones. 1996. A Polymorphic Type System for Extensible Records and Variants. NOTTCS-TR-96-3. University of Nottingham.

Carl A. Gunter, Didier Rémy, and Jon G. Riecke. 1995. A Generalization of Exceptions and Control in ML-like Languages. In Proceedings of the Seventh International Conference on Functional Programming Languages and Computer Architecture, 12-23. FPCA '95. ACM, La Jolla, California, USA. doi:10.1145/224164.224173.

Daniel Hillerström, and Sam Lindley. 2016. Liberating Effects with Rows and Handlers. In Proceedings of the 1st International Workshop on Type-Driven Development, 15-27. TyDe 2016. Nara, Japan. doi:10.1145/2976022.2976033.

Daniel Hillerström, and Sam Lindley. 2018. Shallow Effect Handlers. In 16th Asian Symposium on Programming Languages and Systems (APLAS'18), 415-435. Springer.

Daniel Hillerström, Sam Lindley, Bob Atkey, and KC Sivaramakrishnan. Sep. 2017. Continuation Passing Style for Effect Handlers. In Proceedings of the Second International Conference on Formal Structures for Computation and Deduction. FSCD'17.

Mark P. Jones. Feb. 1992. A Theory of Qualified Types. In 4th. European Symposium on Programming (ESOP’92), 582:287-306 Lecture Notes in Computer Science. Springer-Verlag, Rennes, France. doi:10.1007/3-540-55253-7_17.

Ohad Kammar, Sam Lindley, and Nicolas Oury. 2013. Handlers in Action. In Proceedings of the 18th ACM SIGPLANInternational Conference on Functional Programming, 145-158. ICFP '13. ACM, New York, NY, USA. doi:10.1145/2500365.2500590.

Ohad Kammar, and Matija Pretnar. Jan. 2017. No Value Restriction Is Needed for Algebraic Effects and Handlers. fournal of Functional Programming 27 (1). Cambridge University Press. doi:10.1017/S0956796816000320.

Oleg Kiselyov, Amr Sabry, and Cameron Swords. 2013. Extensible Effects: An Alternative to Monad Transformers. In Proceedings of the 2013 ACM SIGPLAN Symposium on Haskell, 59-70. Haskell '13. Boston, Massachusetts, USA. doi:10.1145/2503778.2503791.

Oleg Kiselyov, and Chung-chieh Shan. 2009. Embedded Probabilistic Programming. In Domain-Specific Languages. doi:10.1007/978-3-642-03034-5_17.

Oleg Kiselyov, Chung-chieh Shan, and Amr Sabry. 2006. Delimited Dynamic Binding. In Proceedings of the Eleventh ACM SIGPLAN International Conference on Functional Programming, 26-37. ICFP '06. Association for Computing Machinery,

Proc. ACM Program. Lang., Vol. 4, No. ICFP, Article 99. Publication date: August 2020. 
New York, NY, USA. doi:10.1145/1159803.1159808.

Daan Leijen. 2005. Extensible Records with Scoped Labels. In Proceedings of the 2005 Symposium on Trends in Functional Programming, 297-312.

Daan Leijen. 2014. Koka: Programming with Row Polymorphic Effect Types. In MSFP'14, 5th Workshop on Mathematically Structured Functional Programming. doi:10.4204/EPTCS.153.8.

Daan Leijen. 2017a. Implementing Algebraic Effects in C: Or Monads for Free in C. Edited by Bor-Yuh Evan Chang. Programming Languages and Systems, LNCS, 10695 (1). Suzhou, China: 339-363. APLAS'17.

Daan Leijen. 2017b. Structured Asynchrony with Algebraic Effects. In Proceedings of the 2nd ACM SIGPLAN International Workshop on Type-Driven Development, 16-29. TyDe 2017. Oxford, UK. doi:10.1145/3122975.3122977.

Daan Leijen. Jan. 2017. Type Directed Compilation of Row-Typed Algebraic Effects. In Proceedings of the 44th ACM SIGPLAN Symposium on Principles of Programming Languages (POPL'17), 486-499. Paris, France. doi:10.1145/3009837.3009872.

Daan Leijen. 2019. Koka Repository. https://github.com/koka-lang/koka.

Sam Lindley, and James Cheney. 2012. Row-Based Effect Types for Database Integration. In Proceedings of the 8th ACM SIGPLAN Workshop on Types in Language Design and Implementation, 91-102. TLDI'12. doi:10.1145/2103786.2103798.

Sam Lindley, Connor McBride, and Craig McLaughlin. Jan. 2017. Do Be Do Be Do. In Proceedings of the 44th ACM SIGPLAN Symposium on Principles of Programming Languages (POPL'17), 500-514. Paris, France. doi:10.1145/3009837.3009897.

Simon L Peyton Jones, and John Launchbury. 1995. State in Haskell. Lisp and Symbolic Comp. 8 (4): $293-341$. doi:10.1007/BF01018827.

Andrew M. Pitts. 1998. Existential Types: Logical Relations and Operational Equivalence. In In Proceedings of the 25th International Colloquium on Automata, Languages and Programming, 309-326. Springer-Verlag.

Atze van der Ploeg, and Oleg Kiselyov. 2014. Reflection without Remorse: Revealing a Hidden Sequence to Speed up Monadic Reflection. In Proceedings of the 2014 ACM SIGPLAN Symposium on Haskell, 133-144. Haskell'14. Göthenburg, Sweden. doi:10.1145/2633357.2633360.

Gordon D. Plotkin, and John Power. 2003. Algebraic Operations and Generic Effects. Applied Categorical Structures 11 (1): 69-94. doi:10.1023/A:1023064908962.

Gordon D. Plotkin, and Matija Pretnar. 2013. Handling Algebraic Effects. In Logical Methods in Computer Science, volume 9. 4. doi:10.2168/LMCS-9(4:23)2013.

Matija Pretnar. Jan. 2010. Logic and Handling of Algebraic Effects. Phdthesis, University of Edinburgh.

Matija Pretnar. Dec. 2015. An Introduction to Algebraic Effects and Handlers. Invited Tutorial Paper. Electron. Notes Theor. Comput. Sci. 319 (C). Elsevier Science Publishers: 19-35. doi:10.1016/j.entcs.2015.12.003.

Matija Pretnar, Amr Hany Shehata Saleh, Axel Faes, and Tom Schrijvers. 2017. Efficient Compilation of Algebraic Effects and Handlers. CW Reports. Department of Computer Science, KU Leuven; Leuven, Belgium. https://lirias. kuleuven . be/retrieve/472230.

Didier Rémy. 1994. Type Inference for Records in Natural Extension of ML. In Theoretical Aspects of Object-Oriented Programming, 67-95. doi:10.1.1.48.5873.

Philipp Schuster, Jonathan Immanuel Brachthäuser, and Klaus Ostermann. 2020. Compiling Effect Handlers in CapabilityPassing Style. In Proceedings of the International Conference on Functional Programming. ACM, New York, NY, USA.

Sebastian Ullrich, and Leonardo de Moura. Sep. 2019. Counting Immutable Beans - Reference Counting Optimized for Purely Functional Programming. In Proceedings of the 31st Symposium on Implementation and Application of Functional Languages (IFL'19). Singapore.

Andrew Wright. 1995. Simple Imperative Polymorphism. In LISP and Symbolic Computation, 343-356.

Nicolas Wu, Tom Schrijvers, and Ralf Hinze. 2014. Effect Handlers in Scope. In Proceedings of the 2014 ACM SIGPLAN Symposium on Haskell, 1-12. Haskell '14. Göthenburg, Sweden. doi:10.1145/2633357.2633358.

Ningning Xie, Jonathan Brachthäuser, Daniel Hillerström, Philipp Schuster, and Daan Leijen. Jul. 2020. Effect Handlers, Evidently. MSR-TR-2020-23. Microsoft Research. Extended version with proofs.

Ningning Xie, and Daan Leijen. Aug. 2020. Effect Handlers in Haskell, Evidently. In Proceedings of the 2020 ACM SIGPLAN Symposium on Haskell. Haskell'20. Jersey City, NJ. doi:10.1145/3406088.3409022.

Yizhou Zhang, and Andrew C. Myers. Jan. 2019. Abstraction-Safe Effect Handlers via Tunneling. Proc. ACM Program. Lang. 3 (POPL). ACM. doi:10.1145/3290318. 